\title{
Electric-field profile and thermal properties in substrate-supported dielectric films
}

\author{
Peter Bloß* and Aimé S. DeReggi ${ }^{\dagger}$ \\ National Institute of Standards and Technology, Gaithersburg, Maryland 20899-8541 \\ Hartmut Schäfer \\ HF-NMR Facility, NSR Center, University of Nijmegen, Toernooiveld 1, NL-6525 ED Nijmegen, The Netherlands
}

(Received 4 January 2000)

\begin{abstract}
The transient pyroelectric response of a dielectric film on a substrate is used to determine both the electricfield profile across the film and its thermal properties. The response is a convolution of the field and temperature profile. A closed-form expression for the transient temperature profile is obtained for a generic model in terms of thermal parameters. This expression allows analysis of the pyroelectric response using an iterative procedure consisting of deconvolution and variation of thermal parameters. Tikhonov regularization is used in the deconvolution with a Honerkamp-Weese self-consistent regularization parameter. Simulations show that, at least in the cases tested, the electric-field profile, the thermal diffusivity and conductivity (and thus the heat capacity, which is their ratio), and the thermal resistance of the dielectric/substrate interface can be determined. Experiments on polyimide films adhered to substrates unambiguously reveal bound negative charge within a small depth of the free surface of virgin samples and also weaker, broader charge distributions injected from the substrate in samples under an applied voltage. The analysis resolves both sharp and broad features and gives thermal properties characteristic of the near-surface region, suggesting that the heat capacity may be significantly higher in this region than in the bulk.
\end{abstract}

\section{INTRODUCTION}

For many years, thermal pulses generated by flashlamps or pulsed lasers have been used to probe electric field/ polarization profiles $^{1-8}$ or thermal properties ${ }^{9-14}$ of electrically insulating materials. The flash method ${ }^{9}$ is widely used to determine the thermal diffusivity of planar samples from the transient temperature rise of the back surface after flashheating the front surface. The thermal pulse (TP) method $^{2-5}$ is used to determine electric-field profiles in charged or polarized dielectrics from the transient pyroelectric response stimulated by pulse-heating a metal electrode coating the incident surface. This response, referred to as the TP response, is taken as the charge $q(t)$ flowing from one electrode to the other in an external short circuit.

The general expression for $q(t)$ for a planar capacitor structure is a convolution integral involving the temperature $T(t, z)$ and the electric field $E(z)$, where $t$ is the time and $z$ the depth from the incident surface. When $T(t, z)$ is known or calculable from the heat conduction equation, the unknown field $E(z)$ is supposed determinable from the measured $q(t)$ by a deconvolution method. For a thermally isolated slab without electrodes, $T(t, z)$ is a cosine Fourier series with exponentially decaying coefficients, ${ }^{15}$ which makes $q(t)$ a sum of decaying exponentials with the same coefficients as those of the cosine Fourier series representing $E(z){ }^{3,4}$ Deconvolution is then reduced to finding coefficients by fitting $q(t)$, and using them to sum the Fourier series representing $E(z)$. The limited number (usually $\leqslant 10$ ) of well-determined coefficients made the analytic resolution lower than the near-surface limit set by the pulse width. Alternative analytic approaches to get around this difficulty have thus been proposed. ${ }^{5}$

Because the time scale of $T(t, z)$ is set by a characteristic time $\tau$ dependent on the thermal diffusivity $D$ and thickness $L\left(\tau=L^{2} / 2 D\right.$ for a thermally isolated $\left.\operatorname{slab}^{15}\right)$, the TP method can be used, like the flash method, to study diffusivity. As recently demonstrated for a voltage-biased dielectric film on a substrate ${ }^{14}$ the diffusivity may be determined with high sensitivity, precision, and speed from $q(t)$ at times $t>\tau$ when $q(t)$ becomes insensitive to the shape of $E(z)$ and proportional to the thickness-averaged temperature $\langle T\rangle$, which decays exponentially. The fitting range in Ref. 14 did not include either very long times, because of the decaying signal, or short times because of the sensitivity of the signal to neglected effects, notably the thermal mass of the incident electrode and the unknown $E(z)$.

In this paper, we investigate the TP problem more thoroughly than previously, using theory, simulation, and experiment. We consider the electrode thermal mass, the heat loss from the incident surface, the thermal resistance at the film/ substrate interface, and the $E(z)$ profile due to inhomogeneous charging of the sample. Inclusion of these effects allows fitting the measured response $q(t)$ over a broad time interval, including short times $(t<\tau)$ where the signal-tonoise ratio is high. The range of application of the TP method is thus extended to include substrate-supported thinfilm structures such as those found in microelectronics and photonics. In such structures, the thermal mass of the electrode will strongly affect the response. The Tikhonov regularization procedure ${ }^{16}$ and Honerkamp-Weese self-consistent regularization parameter ${ }^{17,18}$ were used in a deconvolution procedure that determined the best $E(z)$ for a fixed set of thermal parameters. Successive deconvolutions with systematically adjusted thermal parameters were used to determine the set of parameters that gave a global minimum in the mean square of errors.

The Tikhonov-Honerkamp-Weese procedure is being 


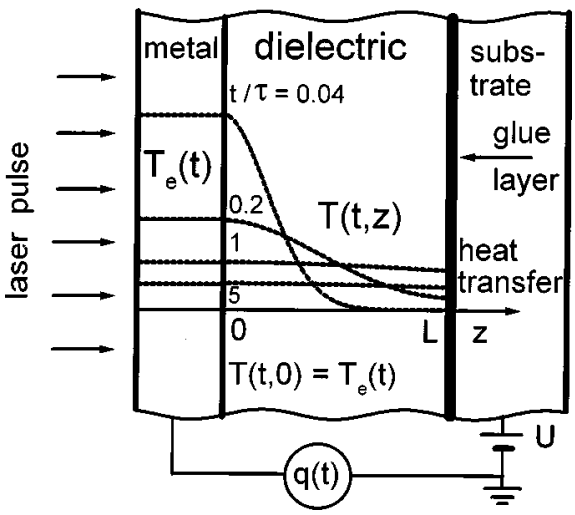

FIG. 1. Model of electrode/dielectric/substrate system heated by thermal pulse. $\quad U$ is bias voltage, $q(t)$ the measured TP response, $T_{e}(t)$ the temperature rise of the electrode, and $T(t, z)$ the temperature rise of dielectric. Dashed curves show $T(t, z)$ for the thermal parameters of Sec. III at indicated reduced times $t / \tau$ [Eq. (8)].

adopted to analyze various types of spectroscopic data ${ }^{19-25}$ but has only recently been applied to thermal methods for determining $E(z)$ profiles, first $^{27-29}$ to the laser-intensitymodulation method ${ }^{30,31}$ (LIMM), a periodic heating method, and here to a thermal pulse method. The TikhonovHonerkamp-Weese procedure finds a good compromise between smoothness in the $E(z)$ profile and low residuals.

The list of materials studied by the TP method includes nonpolar insulating polymers, ${ }^{32}$ polar $^{33,34}$ or ferroelectric polymers, ${ }^{35}$ and ceramics. ${ }^{36}$ These acquire charge or become polarized, generally inhomogeneously, under high applied fields $(>10 \mathrm{~V} / \mu \mathrm{m})$, high temperature $\left(>60^{\circ} \mathrm{C}\right)$, or both. ${ }^{32,37}$ Even polymers with high glass transition temperatures such as polyamides ${ }^{38}$ and polyimides ${ }^{39}$ may acquire charge (or become polarized) when voltage biased. Most of those results were obtained from ring-mounted (i.e., nominally thermally isolated) samples. The experimental results reported here establish the feasibility of determining an inhomogeneous field profile and thermal properties for a substratesupported film.

In Sec. II, the heat conduction model is described, and $T(t, z)$ is given in terms of various parameters included in the model, as the solution to the one-dimensional heat conduction equation. The effects of all parameters on the reference response for a homogeneous field, $q(t) \propto\langle T\rangle$, are shown explicitly and our deconvolution approach is described. The determination of thermal parameters, for both homogeneous and inhomogeneous assumed $E(z)$, is tested in Sec. III. In Secs. IV and V, an actual experiment giving interesting results is described and our algorithms are applied to analysis of the measured responses.

\section{THEORY}

\section{A. Model}

Figure 1 shows our model. The laser pulse is assumed infinitesimally short [and so is represented by a Dirac delta function $\delta(t)$ ]. The pulse heats the metal electrode which then heats the dielectric. Temperatures $T_{e}(t)$ for the electrode (assumed gradient-free for $t \geqslant 0$ ) and $T(t, z)$ for the dielectric are increases relative to a fixed substrate tempera- ture assumed to be zero. We assume perfect contact between electrode and dielectric $\left[T(t, 0)=T_{e}(t)\right]$ and imperfect contact (with thermal resistance $1 / H_{L}$ ) between dielectric and substrate (through a glue layer or simply an interface).

Heat loss to ambient air at the incident surface by convection and radiation is included by using an effective heat transfer coefficient $H_{0}$ with the outgoing flux $H_{0} T_{e}(t) .{ }^{15} \mathrm{~A}$ thermally isolated sample is represented in our model by setting $H_{0}=H_{L}=0$.

\section{B. Heat conduction equation and solution}

The one-dimensional heat conduction equation with $T$ $=T(t, z)$ in our model is ${ }^{15}$

$$
\frac{\partial T}{\partial t}=D \frac{\partial^{2} T}{\partial z^{2}},
$$

with boundary conditions obtained from thermal energy balance. The boundary conditions are

$$
J+H_{0} T+\rho_{e} c_{e} L_{e} \frac{\partial T}{\partial t}=J_{0} \delta(t), \quad z=0,
$$

and

$$
-J+H_{L} T=0, \quad z=L,
$$

where $\rho_{e} c_{e} L_{e}$ is the thermal mass of the electrode unit area. The flux $J=\kappa \partial T / \partial z$ is to be evaluated at the indicated boundaries. Equation (2) with $H_{0}=0, H_{L}=\infty$ and right member replaced by $J_{0} \cos (\omega t)$ was used by Ploss and Bianzano $^{39}$ to analyze the periodic response in the LIMM case. $^{30}$

The solution to Eq. (1) with boundary conditions specified by Eqs. (2) and (3) was obtained by Laplace transformation. ${ }^{15}$ In terms of the reduced depth coordinate $y=z / L, 0 \leqslant y \leqslant 1$, it is

$$
\begin{aligned}
T(t, y)= & \frac{J_{0} L}{\kappa} \sum_{k=0}^{\infty} \frac{x_{k} \cos \left[x_{k}(1-y)\right]+a_{L} \sin \left[x_{k}(1-y)\right]}{-(1 / 2 x) d N(x) / d x} \\
& \times \exp \left(-x_{k}^{2} \frac{t}{2 \tau}\right),
\end{aligned}
$$

where

$N(x)=x\left(a_{0}+a_{L}-r x^{2}\right) \cos (x)+\left[a_{0} a_{L}-\left(1+r a_{L}\right) x^{2}\right] \sin (x)$,

and where the $x_{k}$ are the roots of $N(x)=0$, which must be found numerically in the general case (see below). The dimensionless parameters (called Nusselt numbers $\left.{ }^{40,41}\right) a_{0}, a_{L}$, the ratio $r$ of the thermal mass of the electrode to that of the dielectric, and the diffusion-controlled thermal transit time $\tau$ are

$$
\begin{gathered}
a_{n}=L \frac{H_{n}}{\kappa}, \quad n=0, L, \\
r=\frac{\rho_{e} c_{e} L_{e}}{\rho c L}=\frac{\kappa_{e}}{\kappa} \frac{D}{D_{e}} \frac{L_{e}}{L},
\end{gathered}
$$

and 


$$
\tau=\frac{L^{2}}{2 D}
$$

respectively. The $a_{0}$ and $a_{L}$ are a measure of heat loss. Note that Eqs. (4) and (5) are valid if at least one coefficient $H_{0}$ or $H_{L}$ is greater than zero. For the thermally insulated case $\left(H_{0}=H_{L}=0\right)$, the solution reduces to

$$
\begin{aligned}
T(t, y)= & \frac{J_{0} L}{\kappa}\left[\frac{1}{1+r}+2 \sum_{k=1}^{\infty} \frac{\cos \left[x_{\mathrm{ins}, k}(1-y)\right]}{\left(1+r+r^{2} x_{\mathrm{ins}, k}^{2}\right) \cos \left(x_{\mathrm{ins}, k}\right)}\right. \\
& \left.\times \exp \left(-x_{\mathrm{ins}, k}^{2} \frac{t}{2 \tau}\right)\right]
\end{aligned}
$$

where $x_{\text {ins, } k}$ are now the roots of

$$
N_{\text {ins }}(x)=\sin (x)+r x \cos (x)=0 .
$$

The first term inside the square brackets of Eq. (9), 1/(1 $+r$ ), comes from the residue of the inverse Laplace transform for $x_{\text {ins }, 0}=0$. The factor $J_{0} L / \kappa \equiv T_{\infty}$ is the amplitude of the step jump in temperature for the isolated film $\left(H_{0}=H_{L}\right.$ $=0)$ and $r=0$. For $r=0$, the roots are $x_{\text {ins, } k}(r=0)=k \pi$, where $k \geqslant 1$, and Eq. (9) reduces to the well-known results. ${ }^{4}$ From Eq. (9), for $r>0$, it follows that

$$
T_{\infty}(r>0)=\frac{T_{\infty}}{1+r}=T_{\infty} \frac{\rho c L}{\rho c L+\rho_{e} c_{e} L_{e}},
$$

where $1 /(1+r)$ is the ratio of the thermal mass of the dielectric to the combined thermal mass of the dielectric and electrode. For $H_{0}=0$ and $H_{L}=\infty$, the solution is

$$
\begin{aligned}
T(t, y)= & \frac{J_{0} L}{\kappa} 2 \sum_{k=0}^{\infty} \frac{\sin \left[x_{\text {cont }, k}(1-y)\right]}{\left(1+r+r^{2} x_{\text {cont }, k}^{2}\right) \sin \left(x_{\text {cont }, k}\right)} \\
& \times \exp \left(-x_{\text {cont }, k}^{2} \frac{t}{2 \tau}\right),
\end{aligned}
$$

where $x_{\text {cont }, k}$ are the roots of

$$
N_{\text {cont }}(x)=\cos (x)-r x \sin (x)=0 .
$$

When $r=0$, one obtains the formula given earlier ${ }^{14}$ with the roots $x_{\text {cont }, k}(r=0)=\left(k+\frac{1}{2}\right) \pi$, where $k \geqslant 0$. To our knowledge, the solutions given by Eqs. (4), (9), and (12) are not in the literature.

\section{Response and deconvolution}

\section{The nature of the problem}

The measured charge response $q(t)$ is related to $T(t, y)$ and to $E(y)$, by $^{4,42}$

$$
q(t)=\alpha_{c} C L \int_{0}^{1} T(t, y) E(y) d y,
$$

where $\alpha_{c}=\partial[\ln (C)] / \partial T$ and $C$ is the sample capacitance. When $E(y)$ is replaced by $E$ (with no $y$ dependence), we have the reference case for which $q(t) \sim \int_{0}^{1} T(t, y) d y$ $=\langle T\rangle(t)$.

Equation (14) is a Fredholm integral equation of the first kind with $T(t, y)$ as kernel. The inversion of this type of equation is generally viewed as an ill-posed mathematical problem in the Hadamard sense. ${ }^{43}$ The deconvolution of Eq. (14) with Eq. (4) as kernel when $q(t)$ is a noisy pointsampled function is thus a delicate procedure and any determined function $\hat{E}(y)$ must be viewed as an approximation to the actual $E(y)$. We represent $q(t)$ by

$$
q_{m}^{\sigma}=q\left(t_{m}\right)+\sigma \sigma_{m} \eta_{m}, \quad m=1, \ldots, M,
$$

where $\sigma_{m}=1$ (for $m=1, \ldots, M$ ) is the rms amplitude of noise assumed white and independent of time $t_{m}$ in our simple noise model, the $\eta_{m}$ are Gaussian-distributed random numbers, $\sigma$ is a scaling factor, and $M$ is the number of measuring points at discrete times $t_{m}$ (for $\left.m=1, \ldots, M\right)$.

\section{Our data analysis approach}

For convenience, we rewrite Eq. (14) as

$$
q=\Theta E,
$$

where $\Theta$ is introduced as an integral operator given by

$$
\Theta=\alpha_{c} C L \int_{0}^{1} d y T(t, y) .
$$

In the most general case, the inverse operator $\Theta^{-1}$ does not necessarily exist. The way out is to introduce a pseudoinverse operator $\Theta^{+}$so that the mapping of

$$
\hat{E}=\Theta^{+} q,
$$

the generalized solution, minimizes the distance

$$
d=\|q-\Theta \hat{E}\|
$$

where $\|\cdots\|$ means the Euclidean norm and $\hat{E}$ is the leastsquares solution of Eq. (16). For notational simplicity, we replace $\hat{E}$ by $E$.

Regularization helps to eliminate mathematically admissible but physically unrealistic $E(y)$ by putting an external constraint (smoothness in our case) on the determined $E(y) .{ }^{44-50}$ Regularization approaches include linear and statistical filtering, truncated Fourier analysis, truncated singular-value decomposition, Tikhonov regularization, Miller's regularization, maximum entropy, projection, iteration, stochastic, and other methods. ${ }^{51-55}$

Our algorithm uses the pseudo-inverse approach combined with Tikhonov regularization. The algorithm minimizes

$$
\Psi_{\lambda}(E)=\sum_{m=1}^{M} \frac{d_{m}^{2}}{\sigma^{2}}+\lambda\|\Omega E\|^{2}
$$

where $d_{m}$ are response residuals defined by

$$
d_{m}=q_{m}^{\sigma}-q\left(t_{m}, E\right),
$$

where $q\left(t_{m}, E\right)$ are calculated responses that approximate the measured $q_{m}^{\sigma}$. In Eq. (20), $\sigma$ is the rms noise amplitude [see Eq. (15)]. The parameter $\lambda$ is the regularization parameter. It controls the weight of the regularization term containing the symbol $\Omega$, which is the second-derivative operator. This operator puts curvature in the minimization and thus has a 
smoothing effect on our solutions. For a given $\lambda$, the field profile that minimizes $\Psi_{\lambda}(E)$ is the unique regularized approximate $E_{\lambda}$. In practice, when $\lambda$ is too small, artifacts such as unphysical oscillations may appear in $E_{\lambda}$. When $\lambda$ is too large, $E_{\lambda}$ is oversmoothed and recoverable information is left in the data.

Besides the Honerkamp-Weese method ${ }^{17}$ used here, methods of determining an optimum $\lambda$ include the discrepancy method, ${ }^{44}$ the predictive minimum-mean-square error method, ${ }^{45}$ the Provencher method, ${ }^{56}$ the $L$-curve approach, ${ }^{49}$ and the generalized cross-validation method. ${ }^{57}$ The Honerkamp-Weese method ${ }^{17}$ used here has been tested in Monte Carlo studies and has been applied successfully to many ill-posed problems. ${ }^{17,19-25}$

The optimum value of $\lambda$, symbolized by $\mu$, should minimize the quantity $\langle D\rangle=\left|E_{\text {true }}-F_{\lambda}\right|^{2}$ with respect to $\lambda$, where $E_{\text {true }}$ is the true but unknown distribution and $F_{\lambda}$ is any trial function including $E_{\lambda}$ for a general $\lambda$. Since $E_{\text {true }}$ is not known, we replace $E_{\text {true }}$ by $E_{\mu}$ and solve

$$
\frac{\partial}{\partial \lambda}\left[D\left(E_{\mu}, \lambda, \sigma\right)\right]_{\lambda=\mu}=0
$$

iteratively to obtain self-consistent estimates of optimal $\lambda$ and $\sigma$.

\section{Details for the deconvolution of the TP responses}

Two sets of $N$ grid points $\left\{y_{n}\right\}$ were used, where $N=21$ for the simulations and $N=25-35$ for the deconvolution of the experimental data. Grid-point spacings were determined by a power $\operatorname{law}^{26,27}$ with $y_{1}=0$ at the electrode/dielectric interface and $y_{N}=1$ at the dielectric/substrate interface. The first grid point inside the dielectric was at $y_{2}=b\left(\delta_{\min } / L\right)$, where $b=0.33, \ldots, 0.5$ (see Ref. 27 for details) and $\delta_{\text {min }}$ $=\left(2 D t_{\min }\right)^{1 / 2}$ is the diffusion depth at $t_{\min }$, the earliest time used in the deconvolution. The latest time $t_{\max }$ was taken as the time when the TP response had decreased to one-tenth its maximum. This $t_{\max }$ was large enough to allow $H_{L}$ to be determined. The statistical error bars given in the deconvolution results always represent a $68 \%$ statistical confidence range ( \pm one standard deviation). Details about the deconvolution scheme applicable to both TP and LIMM data may be found in previous LIMM papers. ${ }^{26,27}$ We define the mean square of errors (MSE) of the fit as

$$
\mathrm{MSE}=\frac{1}{M} \sum_{m=2}^{M} d_{m}^{2}
$$

\section{SIMULATION RESULTS}

The simulation results presented here are based on synthetic data and thus are free from experimental artifacts. They test our complex numerical procedure. Experimental results, discussed later, test additionally the adequacy of our model. Properties used in the simulations are representative of a commercial DuPont Kapton ${ }^{\mathrm{TM}}$ polyimide $^{58}(\mathrm{PI})$, namely, $D=7.75 \times 10^{-8} \mathrm{~m}^{2} / \mathrm{s}$ and $\kappa=0.12 \mathrm{~W} / \mathrm{m} \mathrm{K},{ }^{59}$ with a thickness $L=5 \mu \mathrm{m}$. An aluminum electrode was assumed with properties given in Ref. $60, D_{e}=9.79 \times 10^{-5} \mathrm{~m}^{2} / \mathrm{s}$ and $\kappa_{e}$ $=237 \mathrm{~W} / \mathrm{m} \mathrm{K}$, with thickness $L_{e}=(0,100,200) \mathrm{nm}$ corresponding to $r=0.0313$, and $r=0.0625$. A heat transfer coef-

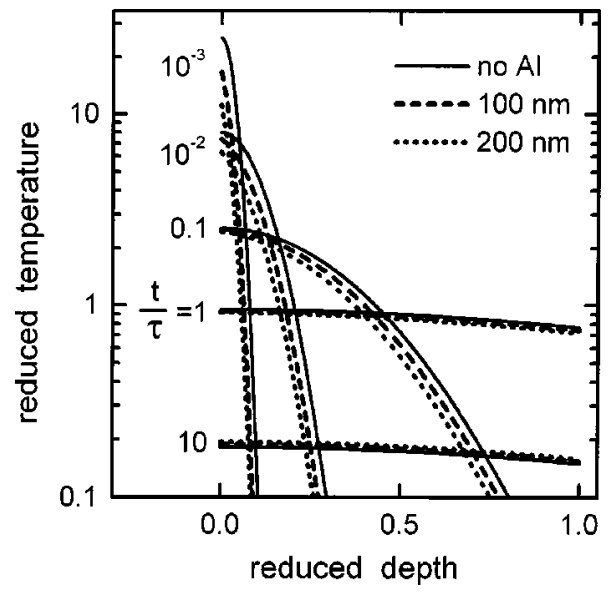

FIG. 2. Reduced temperature profiles $T(t, y) / T_{\infty}$ vs $y$ from Eq. (4), for different $t / \tau$, for realistic $H_{L}=10^{4} \mathrm{~W} / \mathrm{m}^{2} \mathrm{~K}$, or $a_{L}$ $=0.4167$ and electrode thicknesses shown $\left(T_{\infty}=J_{0} L / \kappa, \tau\right.$ $=L^{2} / 2 D$ [Eq. (8)]).

ficient $H_{0}=15 \mathrm{~W} / \mathrm{m}^{2} \mathrm{~K}$ was used, ${ }^{41,61}$ typical of air, yielding $a_{0}=6.25 \times 10^{-4}$. A previously determined $H_{L}$ $=10^{4} \mathrm{~W} / \mathrm{m}^{2} \mathrm{~K}$ corresponding to $a_{L}=0.417$ was used, ${ }^{26,27}$ as representative of the adhesive used in the measurement described in Sec. V.

To test the determination of thermal parameters, synthetic TP responses were constructed by adding white Gaussian noise to ideal responses calculated numerically for three cases, homogeneous $E$ and two model inhomogeneous $E(y)$, for materials with the thickness and thermal parameters given above. The minimum rms amplitude of the added noise was $0.3 \%$ of the peak value, representative of the signal-to-noise ratio obtained in the measurements described later in this paper, using 50-shot signal averaging. Higher noise was used when testing procedural robustness.

The responses were evaluated at 200 points per decade of time, equidistant on a logarithmic time scale. The fitting range started at $t_{\min }=10^{-3} \tau=161 \mathrm{~ns}$, a time compatible with the laser pulse ( $99.5 \%$ of energy delivered in $150 \mathrm{~ns}$ ). The range of the analysis was covered by 830 points.

In the abscissas of the following figures, actual times for realistic experimental conditions are given in addition to reduced times. This is to convey a feeling for actual experimental times.

\section{A. Transient temperature profiles}

Transient temperature profiles were obtained from Eq. (4) and the roots $x_{k}$ of Eq. (5). The roots reflect the boundary conditions and vary smoothly in the range

$$
x_{\text {ins }, k} \leqslant x_{k} \leqslant x_{\text {cont }, k},
$$

where $x_{\text {ins, } k}$ is a lower limit for the thermally insulated case and $x_{\text {cont, }, k}$ is an upper limit for the perfectly thermally contacted case. For $r=0$ (no electrode), $x_{\text {ins }, k}=k \pi$ and $x_{\text {cont }, k}$ $=\left(k+\frac{1}{2}\right) \pi$, where $k=0,1,2, \ldots$. From the decaying exponential terms of Eq. (4), the number of roots significantly affecting $T(t, y)$ decreases with time. For $t>\tau, T(t, y)$ becomes dominated by the slowest-decaying term determined by the lowest root.

Figure 2 shows semilogarithmic plots of the reduced temperature $\left[T(t, y) / T_{\infty}\right]$ vs the reduced depth $y=z / L$ (tempera- 


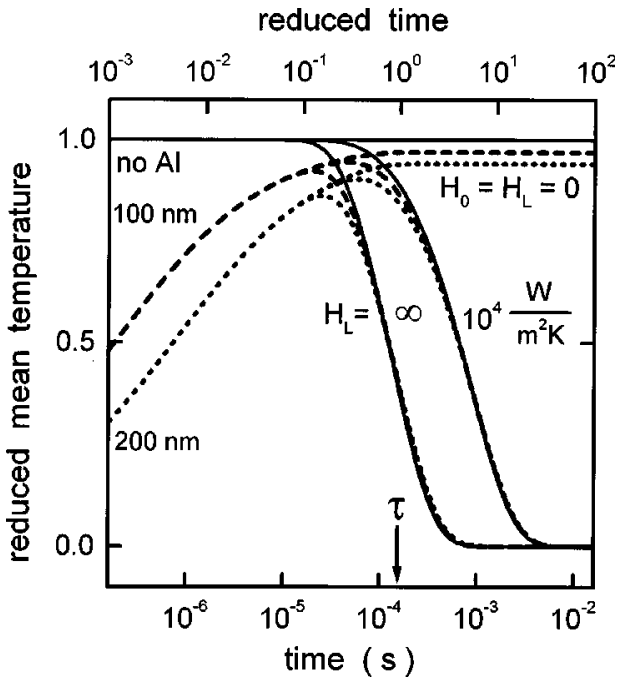

FIG. 3. Reduced mean temperature $\langle T\rangle / T_{\infty}$ vs $\log _{10} t$ for $H_{L}$ and electrode thicknesses shown. Note shift to longer $t$ for decreasing $H_{L}$. Note $\lim \left(\langle T\rangle / T_{\infty}\right)=1 /(1+r)$ for $t \rightarrow \infty$, and $H_{0}=H_{L}=0$ [see Eq. (11)]. From data in Sec. III, $1 /(1+r)=0.970$ and 0.941 for 100 and $200 \mathrm{~nm} \mathrm{Al}$, respectively.

ture profile) at various reduced times $t / \tau$ for the mathematical limit of zero electrode thickness $(r=0)$ labeled "no Al", and two realistic thicknesses (100 and $200 \mathrm{~nm}$ ). As defined previously, $T_{\infty}$ is the asymptotic limit of the temperature rise for the thermally isolated sample and for $r=0$.

\section{B. Mean temperature}

For samples with $y$-independent $E$, namely, uniformly polarized samples or charge-free samples not subject to charge injection, $q(t)$ is proportional to the mean temperature $\langle T\rangle$ $=\int_{0}^{1} T(t, y) d y$, as previously indicated [see Eq. (14)]. This useful reference response is discussed presently.

Figure 3 shows the reduced mean temperature $\langle T\rangle / T_{\infty}$, plotted against $\log _{10} t$, for the same thicknesses as before (Fig. 2) and several contacting conditions specified by $H_{0}$ and $H_{L}$. The $\log _{10} t$ plot conveniently shows $\langle T\rangle / T_{\infty}$ over many orders of magnitude. For $r=0$ and $H_{0}=H_{L}=0$, $\langle T\rangle / T_{\infty}$ rises instantaneously (for a $\delta$-function pulse) from zero to the ideally constant maximum value of unity. The electrode effects are seen by comparing the " $100 \mathrm{~nm}$ " and "200 nm" curves to the "no Al" curve. The electrode effects are manifested by a gradual rise of $\langle T\rangle / T_{\infty}$ to a peak value smaller than unity. For $H_{L}>0$ and $r=0$ ("no $\mathrm{Al}$ "), $\langle T\rangle / T_{\infty}$ shows a decay from unity starting at a time that depends on $H_{L}$. This decay occurs at the earliest times ( $t$ $\approx \tau$ ) for perfect thermal contact $\left(H_{L}=\infty\right)$, and at later times for finite $H_{L}$. For $H_{L}>0$ and $r>0,\langle T\rangle / T_{\infty}$ rises to an $r$-dependent peak value. The decay of $\langle T\rangle / T_{\infty}$ from the peak approaches an exponential for $t>\tau$ but the semilogarithmic slope actually depends on $D, H_{L}$, and also $r$. This has implications for the common practice of determining $D$ from this slope, as discussed in Sec. III E 2 b. For $H_{0}=H_{L}=0$, $\langle T\rangle / T_{\infty}$ approaches an $r$-dependent limiting value for $t \gg \tau$.

In summary, the electrode has thermal buffering effects. It absorbs thermal energy during the short thermal pulse but only slowly transfers it to the dielectric (transfer limited by the diffusivity of the dielectric). The time-dependent sharing
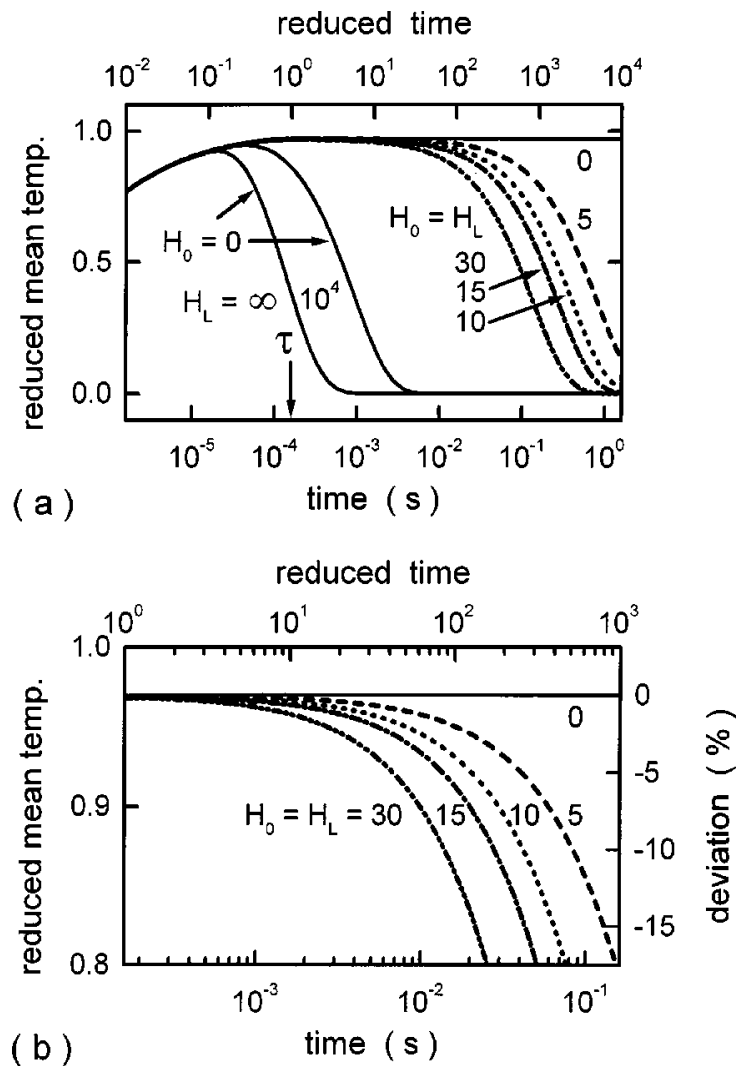

FIG. 4. $\langle T\rangle / T_{\infty}$ vs $\log _{10} t$ for various $\left(H_{0}, H_{L}\right)$ with $100 \mathrm{~nm}$ Al. (a) Solid curves, front thermally isolated $\left(H_{0}=0\right)$; rear, substrate contacted. Dotted and dashed curves, free-standing sample in air. (b) Expanded graph of dotted and dashed curves in (a). Vertical scale at right is the error relative to plateau value for $H=H_{0}$ $=H_{L}=0$. Values for $H_{0}$ and $H_{L}$ in W/m $/ \mathrm{m}^{2} \mathrm{~K}$. Range of $a=L H / \kappa$ is $0-1.25 \times 10^{-3}$.

of energy between the electrode and the dielectric results in delayed entry of heat into the dielectric, with substantial effects on the diffusion process over a broad range of time.

\section{Front-surface heat loss}

Figure 4 shows the effects of heat loss from the incident surface to atmospheric air. The reduced mean temperature is shown for various $\left(H_{0}, H_{L}\right)$ combinations for $100 \mathrm{~nm} \mathrm{Al}$. As discussed in Sec. II A, for small $T_{e}$ (a few kelvin typically), $H_{0}$ includes loss by convection and radiation.

The combinations $(0, \infty)$ and $\left(0,10^{4}\right)$ represent realistic limits (solid curves) for a substrate-supported dielectric. The remaining combinations are for a free-standing sample in air with $H=H_{0}=H_{L}=(5,10,15,30) \mathrm{W} / \mathrm{m}^{2} \mathrm{~K}$, representative of actual values. ${ }^{41}$ As expected, for the substrate-mounted dielectrics considered here, $H_{L} \gg H_{0}$, and the small heat transfer into air has a minor effect at all experimentally relevant times. Figure 4(b) shows $\langle T\rangle$ at long times. The scale on the $y$ axis on the right side of the graph gives the percentage deviation from the maximum reduced mean temperature. For the assumed dielectric thickness of $5 \mu \mathrm{m}$, the effect on $q(t)$ of neglecting the heat transfer to the surrounding air $(H$ $\leqslant 30 \mathrm{~W} / \mathrm{m}^{2} \mathrm{~K}$ ) is smaller than $1 \%$ up to $t \sim 1 \mathrm{~ms}$. In all further simulations and data analysis, we shall use $H_{0}$ $=15 \mathrm{~W} / \mathrm{m}^{2} \mathrm{~K}$ as a realistic value. , $^{81,61}$ 


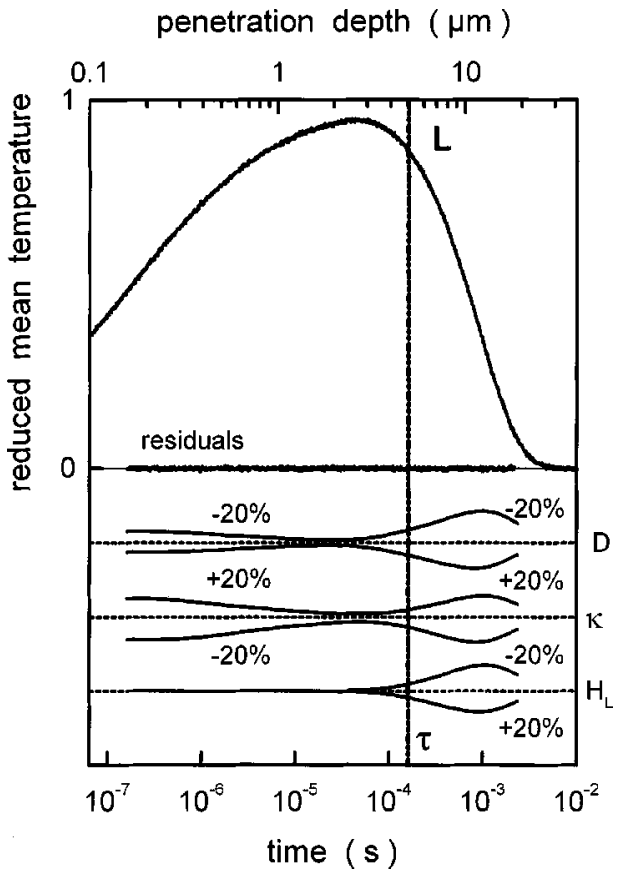

FIG. 5. Uppermost curve: $\langle T\rangle / T_{\infty}$ vs $\log _{10} t$ for thermal parameters of Sec. III, $0.3 \%$ noise. Next curve down: residuals after fitting $\langle T\rangle / T_{\infty}$ by variation of thermal parameters. Lowest three curves: off-minimum residuals obtained by setting the parameter indicated at right $\pm 20 \%$ off its variationally optimal value. Shifted zero baselines shown by horizontal dotted lines.

\section{Thermal parameters for homogeneous field}

Figure 5 shows how the parameters $D, \kappa$, and $H_{L}$ affect $q(t)$ in the case when $E$ is homogeneous and therefore $q(t) \propto\langle T\rangle$. The simulated $q(t) \propto\langle T\rangle$ (uppermost curve) was constructed by adding $0.3 \%$ noise to $\langle T\rangle$ in Fig. 3 for $100 \mathrm{~nm}$ $\mathrm{Al}$, with $\alpha_{C} C L=1 \mathrm{C} \mathrm{m} / \mathrm{V} \mathrm{K}$ in Eq. (14) and $T_{\infty}=1 \mathrm{~K}$. This $q(t)$ was fitted using Eqs. (4) and (5) with $D, \kappa$, and $H_{L}$ treated as adjustable parameters and $H_{0}=15 \mathrm{~W} / \mathrm{m}^{2} \mathrm{~K}$. Note that any two of the three parameters $D, \kappa$, and $\rho c$ may be treated as independent, the third being determined by the definition $D=\kappa /(\rho c)$. The fit gave the correct $D, \kappa$, and $H_{L}$ at a unique MSE minimum. The residuals (next curve down) form a noisy line on top of the zero baseline and also show the range fitted.

The other curves (shown shifted in steps of -0.2 units) are "off-minimum" residuals obtained by setting one parameter at a time (the one indicated at the right of each shifted baseline) $\mp 20 \%$ off its posited value. The influence of each parameter is seen in a systematic, time-dependent deviation of the residuals from each baseline. The range of influence of each parameter is consistent with the boundary conditions [Eqs. (2) and (3)] that come into play at short ( $t$ $\ll \tau)$ and long $(t \geqslant \tau)$ times, respectively.

A strong influence of $D$ and $H_{L}$ is expected at longer times when heat reaches the back surface. A strong influence of $\kappa$ (and thus $D$ ) may be expected at short times $(t \ll \tau)$ too from the boundary condition at $z=0$ in Laplace space, ${ }^{15}$

$$
-\frac{d v}{d z}+\left(r \xi^{2}+a_{0}\right) v=\frac{J_{0}}{\kappa},
$$

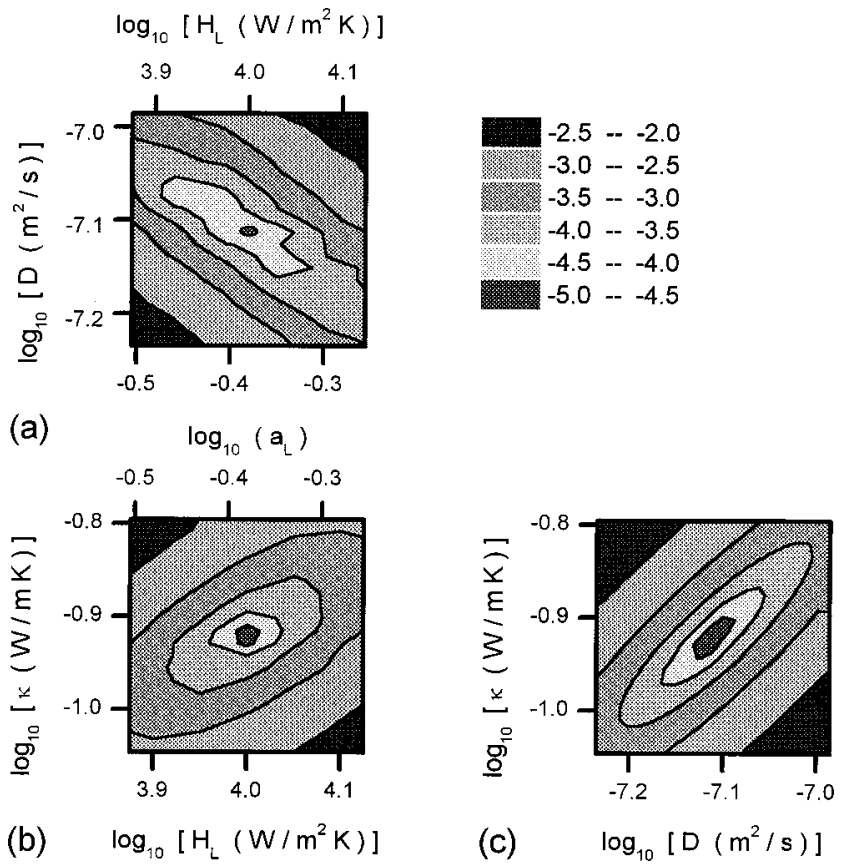

FIG. 6. Constant-MSE contour plots obtained when fitting $\langle T\rangle / T_{\infty}$ in Fig. 5 by variation of two out of three thermal parameters $\left(D, \kappa\right.$, and $H_{L}$ ) with the third kept at optimal value. (a) $D$ and $H_{L}$ were varied; (b) $\kappa$ and $H_{L}$ were varied; and (c) $\kappa$ and $D$ were varied. In (a) and (b), $a_{L}$ from Eq. (6) is shown as well as $H_{L}$. The global MSE minimum (point where $D, \kappa$, and $H_{L}$ have their optimal values) lies at center of each plot. Inclination of ellipsoidal contour indicates correlation of parameters varied.

where $v$ is the Laplace transform of $T$ and $\xi^{2}=L^{2} p / D$ with $p$ as Laplace coordinate. As $D$ is contained in $r$, it affects the temperature profiles near $z=0$. Thus, for $r>0, D$ may be determined at short times (from the calorimetric effects of the electrode) or at long times.

Variation of the three parameters $\left(D, \kappa, H_{L}\right)$ over a wide range (on a $\log _{10}$ scale, $D$ and $\kappa$ were each varied by $\pm 30 \%$ and $H_{L}$ was varied by $\pm 100 \%$ from their posited values) produced a unique minimum of the MSE at the posited values of the parameters. Figure 6 shows MSE contours obtained by varying only two parameters at a time. Only the range of variation of $\pm 12.5 \%$ on the $\log _{10}$ scale is shown. The center of each plot corresponds to the global minimum. The two-dimensional contour plots give graphical evidence for the reliability of the fit (uniqueness of the minimum) and the correlation between the parameters.

The ellipsoidal shape of the contour plots in Fig. 6 indicates different sensitivity of the MSE to different parameters. The statistical error, the ratio between the standard deviation and the known values, is $\approx 1 \%$ for $D, \approx 2 \%$ for $\kappa$, and $\approx 5 \%$ for $H_{L}$. The inclination of the principal axes of the ellipsoids indicates the degree of correlation between the parameters. In the absence of correlation, the principal axes would coincide with the two coordinate axes. Correlation may be expected from Eq. (5) where $N(x)$ contains terms that contain more than one thermal parameter. Additional comments may now be made about each contour plot.

\section{1. $D$ and $H_{L}$}

Figure 6(a) shows the contour plot obtained when $D$ and $H_{L}$ are varied while $\kappa$ is set to its correct value. The angle of 
inclination of the closed contours is negative, because increases in both $D$ and $H_{L}$ cause a stronger decay of $q(t)$ $\propto\langle T\rangle$ (see also Sec. IIIE $2 \mathrm{~b}$ below). The fitting procedure gave the same minimum when the noise was increased from $0.3 \%$ to $1 \%$ and then to $3 \%$.

\section{2. $\kappa$ and $H_{L}$}

Figure 6(b) shows MSE contours when $\kappa$ and $H_{L}$ are varied. The angle of inclination is positive because at long times $(t>\tau)$ the ratio $h_{L}=H_{L} / \kappa$ determines the boundary condition at $z=L$ [Eq. (3)], meaning an increase of $H_{L}$ has the same effect as a decrease of $\kappa$. The robustness of the procedure against added noise is at least as good as that found when $D$ and $H_{L}$ were varied.

\section{3. $D$ and $\kappa$}

Figure 6(c) shows the MSE contours obtained when $D$ and $\kappa$ are varied. The angle of inclination is $+45^{\circ}$ because, for a given value of $\rho c$, an increase of $\kappa$ has the same effect as a decrease of $D$.

We note that the electrode effects on $q(t)$ were sufficiently large (for $100 \mathrm{~nm} \mathrm{Al}$ ) to allow $D$ and $\kappa$ to be found even when the fitting range was deliberately restricted to $t$ $<\tau$. Finding a unique minimum in this case required that the added noise level not exceed $0.3 \%$.

\section{E. Response and deconvolution for inhomogeneous $E(y)$}

\section{Electrode effects in deconvolution}

Figures 7(a) and 8(a) show the two model $E(y)$ profiles (solid lines) for the simulations: the first, in Fig. 7(a), is positive with a steplike decrease; the second, in Fig. 8(a), is negative with a displaced peaklike shape. Their absolute mean values $\left\langle E_{\text {step }}\right\rangle$ and $\left\langle E_{\text {peak }}\right\rangle$, where $\langle E\rangle=\int_{0}^{1} E(y) d y$, are set equal to each other and are shown by the dashed lines. In this section, we assume the thermal parameters are known in order to discuss electrode effects in the deconvolution designed to determine $E(y)$. TB responses $q(t)$ were therefore calculated from Eq. (14) with $T(t, y)$ given by Eqs. (4) and (5) with the known values of the thermal parameters. The electrode effects are then included or excluded by the value assigned to $r$. In this section, we use $r=0$ (no electrode) or $r=0.03127(100 \mathrm{~nm} \mathrm{Al})$.

Figures $7(\mathrm{~b})$ and $8(\mathrm{~b})$ show separate sets of three calculated $q(t)$. In each set, two are for the assumed inhomogeneous $E(y)$. The noiseless solid curve is for no electrode $(r=0)$ and the noisy solid curve is for $100 \mathrm{nmAl}(r$ $=0.03127$ ). The third curve in each set, the dashed curve, is for homogeneous $E$ equal to the common $\langle E\rangle$, for, again, $100 \mathrm{~nm} \mathrm{Al}$. Since absolute amplitudes of $q(t)$ are not of concern in this paper, the $q(t)$ axes are graduated in arbitrary units corresponding to $\alpha_{C} C L=1 \mathrm{C} \mathrm{m} / \mathrm{V} \mathrm{K}$ in Eq. (14). Comparison of the two solid curves for each $E(y)$ reveals the effects of the electrode on $q(t)$ in the two cases. Comparison of the noisy solid curve and the dashed curve for each $E(y)$ reveals the effects of the inhomogeneity in $E(y)$.

The effects of the electrode on $q(t)$ are clearly stronger for the steplike $E(y)$ than for the peaklike $E(y)$. This follows from the convolution integral [Eq. (14)], from which it may be seen that $\int_{0}^{\eta} E(y) d y$, where $\eta$ represents a shallow
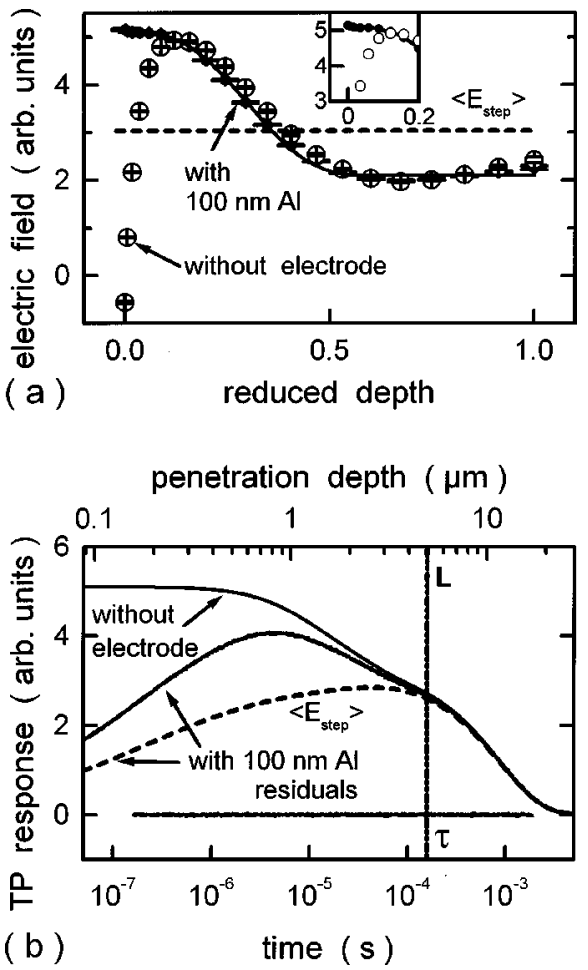

FIG. 7. Electrode effects in deconvolution. (a) Solid curve, "steplike" model $E(y)$; dashed line, mean value $\left\langle E_{\text {step }}\right\rangle$. Filled circles on solid curve, $E(y)$ obtained by deconvolution of TP response when $100 \mathrm{~nm} \mathrm{Al}$ is assumed in both simulated response and in deconvolution (statistical error bars smaller than filled circle diameter). Open symbols (in inset) and dotted line, $E(y)$ obtained by deconvolution when $100 \mathrm{~nm} \mathrm{Al}$ is assumed in simulated response, but not in deconvolution (simulates neglecting electrode effects in data analysis). (b) TP responses calculated from model $E(y)$ and residuals.

depth, is larger for $E_{\text {step }}$ than for $E_{\text {peak }}$, and the fact that electrode effects on $T(t, y)$ are strongest at short times (Fig. 3).

Figures 7(a) and 8(a) also show points (filled and open circles with small error bars) representing $E(y)$ obtained from the simulated $q(t)$ in Figs. 7(b) and 8(b) by deconvolution. Residuals are shown in Figs. 7(b) and 8(b). They show the fitting range as well as the quality of the fit.

The filled circles (one is labeled "with $100 \mathrm{~nm} \mathrm{Al")} \mathrm{are}$ obtained when electrode effects are included in both the calculation of the simulated $q(t)$ and in the deconvolution. These points reproduce the model $E(y)$ (solid lines) quite accurately. The region near $y=0$ is shown in the insets at the top of Figs. 7(a) and 8(a).

The open circles are obtained when electrode effects are included in the calculation of the simulated $q(t)$ but not in the deconvolution [accomplished by resetting $r$ in Eqs. (4) and (5)]. These points reproduce the model $E(y)$ well at large $y$ but show a sharply deviant dip near $y=0$. This dip illustrates the large errors (not apparent in the residuals, which remain small) that may result if electrode effects present in acquired data are not considered in the data analysis. The dip comes from associated error in determining the peak surface temperature. The dip does not appear for the peaklike $E(y)$ because this $E(y)$ is small or zero near $y$ $=0$. 

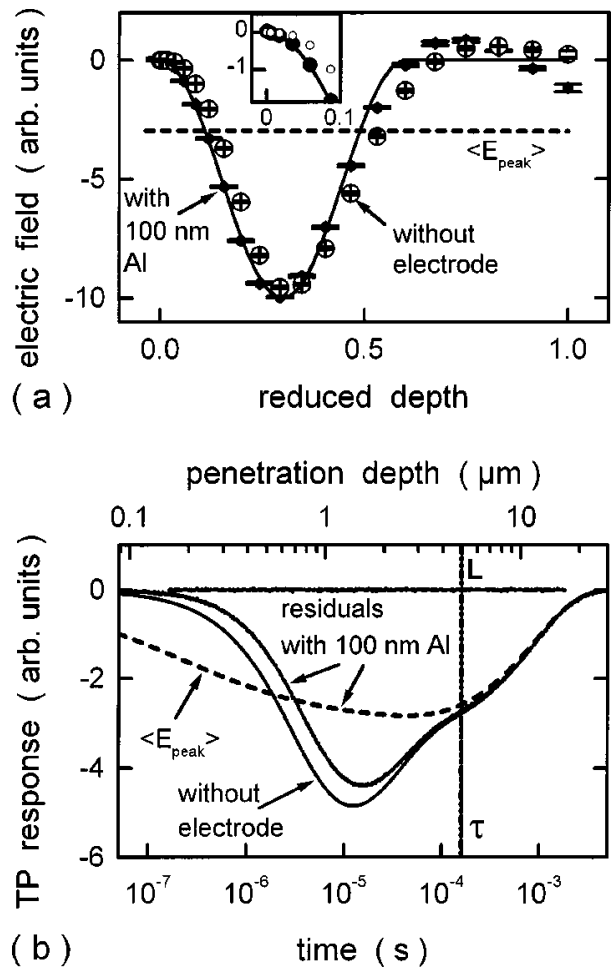

FIG. 8. Same as Fig. 7, but for the "peaklike" model $E(y)$.

The above simulations suggest that a deviation in $q(t)$ at small $t$ (due to an inaccurate $r$ or $\kappa$ ) could be compensated by the creation of a deviation in $E(y)$ at small $y$ with only subtle effects on the residuals. The minimization procedure would make the residuals small at short times where $q(t)$ is most affected by $E(y \approx 0)$, but may not be able to keep the residuals as small at long times. Similar comments apply to deviations in $\kappa$. Finally, the importance of such effects and the diagnostic value of residuals depend on the shape of $E(y)$, which is unknown a priori in actual experiments.

\section{Thermal parameters for nonuniform $E(y)$}

In determining $E(y)$ from actual samples, the thermal parameters are generally unknown, or known only approximately. Parameters in manufacturer data sheets may not be strictly applicable because of possibly different measuring conditions. There is thus a need to investigate the feasibility of determining the in situ thermal parameters in the process of determining $E(y)$. Feasibility cannot be proven generally, but is illustrated here for our two model $E(y)$.

We again used TP responses calculated from the $E(y)$ models shown in Figs. 7(a) and 8(a) for fixed thermal parameters and $100 \mathrm{~nm} \mathrm{Al}$, and let the analysis procedure determine the thermal parameters along with $E(y)$. For each model $E(y)$, the combined deconvolution and variational procedure yielded a global minimum but, now, secondary minima were also found. Still, the global minimum gave satisfactory $E(y)$, as shown by filled circles in Figs. 7(a) and 8(a), together with thermal parameter values in agreement with the known ones. As before, the residuals showed no systematic deviations from the zero baseline, as in Figs. 7(b) and $8(\mathrm{~b})$. The fact that $D, \kappa, H_{L}$, and $E(y)$ could be found simultaneously for both model $E(y)$ suggests that this may be possible also for at least some a priori unknown $E(y)$ profiles.

a. Uniqueness of $E(y)$ and values of thermal parameters. The existence of secondary minima in the MSE, albeit shallower ones than the correct one, for each of our two model $E(y)$ raises questions about the possibility that the combined deconvolution/variation procedure could yield a false global minimum in a general experimental situation. We cannot rule out this possibility. So we suggest that, if multiple minima are found, the principle of parsimony ${ }^{57}$ should be used as a guide for evaluating them. This means selecting the deepest one that gives the smoothest $E(y)$.

In our simulations, we obtained spurious oscillations in $E(y)$ if any of the thermal parameters took on values differing significantly from those assumed in calculating $q(t)$. A plausible mathematical formulation of the principle of parsimony is based on the norm of $E(y),\|E(y)\|$ $=\left[\int_{0}^{1} E^{2}(y) d y\right]^{1 / 2}$, which increases if the amplitude of the oscillations increases. The solution $E(y)$ with the smallest norm has the smallest oscillations and thus may be considered as the most probable one. The thermal parameters accompanying the most probable $E(y)$ likewise may be considered the most probable ones. We emphasize that the selfconsistency method ${ }^{17,18}$ itself provides only one $E(y)$ for one set of thermal parameters.

$b$. Elaboration on simple determinations of $D$. Simple determinations of $D$ involve fitting experimental $q(t)$ at long times $(t>\tau)$ where $q(t)$ approaches a simple exponential decay according to both our model and a simpler one. ${ }^{14}$ Figure 9(a) shows the $\log _{10}|q(t)|$ vs $t$ calculated using Eqs. (4), (5), and (8) for a 5- $\mu \mathrm{m}$-thick PI film with $200 \mathrm{~nm} \mathrm{Al} \mathrm{elec-}$ trodes for three different $E(y)$ profiles and two different $H_{L}$. Figure 9(b) shows the same results on a log-log plot. The effects of the $E(y)$ profile are seen in the amplitude and position of the peak response in the time range $t<\tau$. Electrode effects in the same range are seen in the curve for uniform $E(y)$ as deviations from unity response, but these effects extend to longer times as quantified below. In Fig. 9(a), curves with the same $H_{L}$ approach parallel lines at long times $(t>\tau)$ specified by

$$
\log _{10}|q(t)|=w-s t
$$

where both the semilogarithmic slope $s$ and intercept $w$ are positive. From Eq. (4) in the limit $t \rightarrow \infty$, we obtain $s=x_{0}^{2} D / L^{2}$, where the root $x_{0}\left[0 \leqslant x_{0} \leqslant \pi / 2\right.$ from Eq. (5)] contains the electrode and interfacial effects. When the electrode thermal mass is neglected and ideal thermal contact $\left(H_{L}=\infty\right)$ is assumed, as in the simpler model, ${ }^{14}$ we obtain $x_{0}=\pi / 2$ and recover the result of the simpler model, $D$ $=4 s L^{2} / \pi^{2}$. We can now estimate errors in $D$ associated with neglecting electrode effects and imperfect thermal contact for the conditions used in Fig. 9.

From Eq. (5), neglecting a 100-nm-thick electrode reduces $x_{0}$ by around $3.2 \%$ for $H_{L}=\infty$. This reduces $s$ and leads to an error in $D$ of $6.4 \%$. Imperfect thermal contact also reduces $x_{0}$. For an assumed $H_{L}=10^{5} \mathrm{~W} / \mathrm{m}^{2} \mathrm{~K}$ consistent with our results, we find that $x_{0}$ is reduced by about $18 \%$ so that the error in $D$ would be about $36 \%$.

In Fig. 9(a), we note that, as $H_{L}$ decreases, $q(t)$ remains exponential for $t>\tau$, but the semilogarithmic slope $s$ 

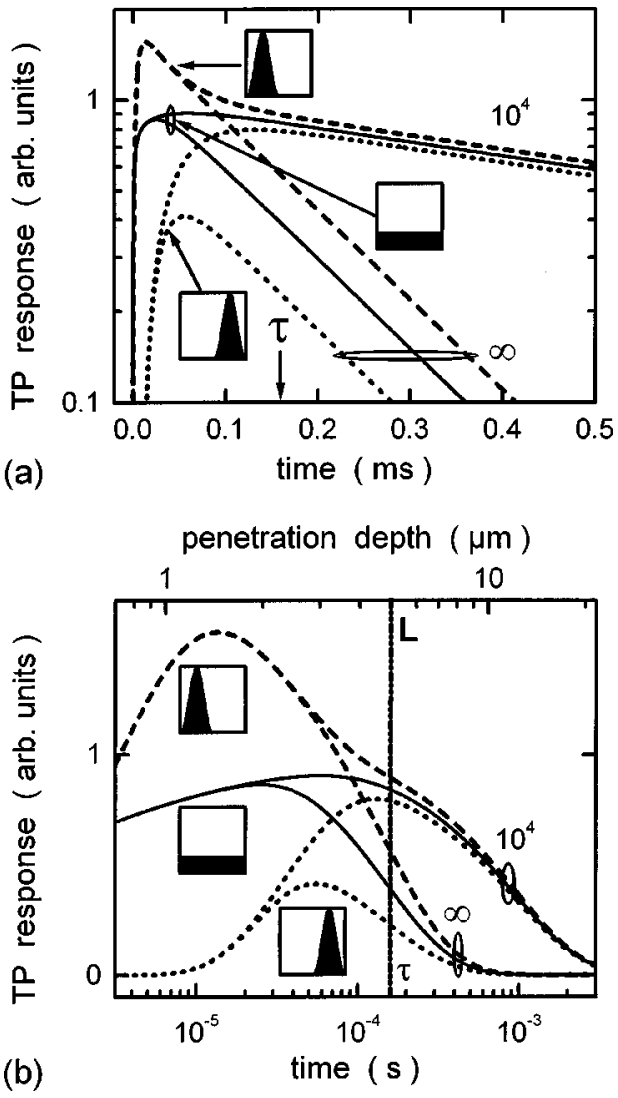

FIG. 9. (a) $\log _{10}|q(t)|$ vs $t$ for a $y$-independent $E$ and two peaklike $E(y)$ with the common mean value $\langle E(y)\rangle$, for $200 \mathrm{~nm} \mathrm{Al}$ electrode and 5- $\mu$ m-thick PI film with thermal parameters of Sec. III. Curves are parallel for $t>\tau$, but are shifted along time axis according to $E(y)$. Numbers $10^{4}$ and $\infty$ refer to $H_{L}$ in $\mathrm{W} / \mathrm{m}^{2} \mathrm{~K}$. Note $H_{L}$ dependence of slope at $t \gg \tau$ with maximum slope for $H_{L}=\infty$. (b) Same as (a), except that plot is $q(t)$ vs $\log _{10}(t)$. Note dependence of $q(t)$ on $E(y)$ for $t<\tau$ and shift of decay for $t>\tau$ when $H_{L}$ goes from $\infty$ to $10^{4} \mathrm{~W} / \mathrm{m}^{2} \mathrm{~K}$.

$=s\left(D, H_{L}\right)$ becomes increasingly dependent on $H_{L}$. As seen in Fig. 9(b) and also in Figs. 3 and 4, a decrease in $H_{L}$ causes a shift of the decaying part of $q(t)$ to longer times, suggesting that $H_{L}$ determines $w$, i.e., $w=w\left(H_{L}\right)$. We suggest that $D$ and $H_{L}$ may be estimated simply from $s$ and $w$ by obtaining simultaneous solutions to the equations $s=s\left(D, H_{L}\right)$ and $w=w\left(H_{L}\right)$, which is possible only for homogeneous $E$. As seen in Fig. 9(a), for inhomogeneous $E(y)$, the linear parts of $\log _{10}|q(t)|$ for the three $E(y)$ are parallel, meaning that $w$ depends on $E(y)$. Thus, for an inhomogeneous $E(y), D$ and $H_{L}$ may be determined simultaneously only if $E(y)$ is known a priori (or is determined by our methods).

$c$. Comments on determining $\kappa$. The deconvolution procedure also yields a best-fitting value of the thermal mass ratio $r$. Using published values of $\rho$ and $c$ for the Al electrode and the known thickness of the electrode and the dielectric, one can deduce a value for $\rho c$ of the dielectric. From this and the value of $D$ obtained from the deconvolution, the thermal conductivity $\kappa$ of the dielectric can be deduced from $\kappa$ $=D \rho c$. The effects of a $\pm 15 \%$ change in $\kappa\left(\kappa_{a}=\kappa\right.$ $\pm 0.15 \kappa)$ in the deconvolution results are shown in Figs. 10 (a) and 10 (b) for a 5 - $\mu$ m-thick film with a 100 -nm-thick Al electrode and the model $E(y)$ in Figs. 7(a) and 8(a). For
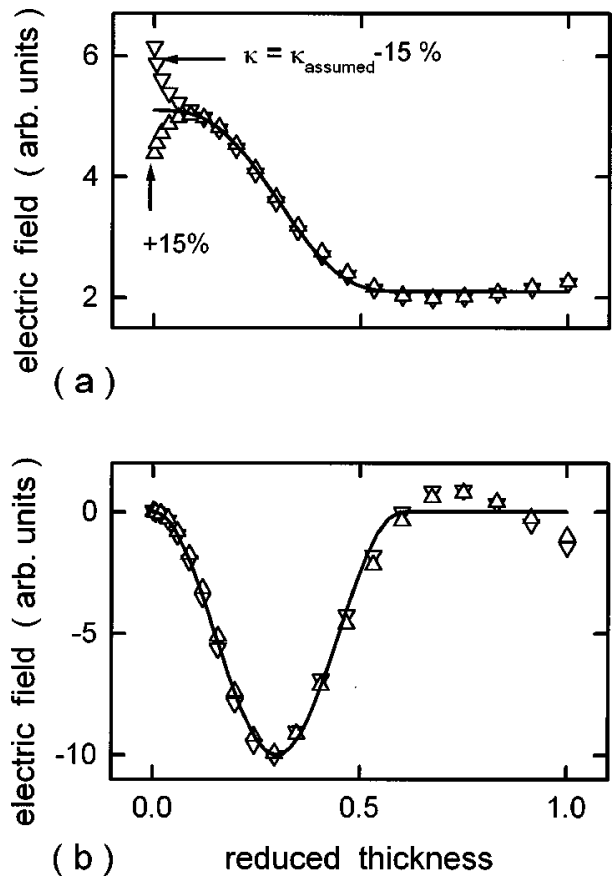

FIG. 10. Influence of $\kappa$ deviation on $E(y)$ obtained by deconvolution for (a) steplike $E(y)$ and (b) peaklike $E(y)$. Deconvolution with $\kappa=\kappa_{a}-15 \%(\nabla)$ and with $\kappa=\kappa_{a}+15 \%(\triangle)$ with constant $h_{L}=H_{L} / \kappa=H_{L, a} / \kappa_{a} \quad\left(\kappa_{a}\right.$ accepted value from Sec. III $)$. Statistical error bars are smaller than symbols and are omitted for clarity. For (a), large deviations (solid lines) are due to peak in $E(y)$ near $y=0$ sensitizing short- $t$ range where $\kappa$ has strong influence (see Fig. 6). For $(b)$, no deviations because $E(y \approx 0) \approx 0$ desensitizes short $t$ range of influence of $\kappa$.

these deconvolutions the value of $H_{L}$ was adjusted so that $h_{L}=H_{L} / \kappa=H_{L, a} / \kappa_{a}$ was constant, because the boundary condition at $z=L\left[\right.$ Eq. (3)] depends on $h_{L}$.

Large deviations in the determined $E(y)$ are seen near the front side in Fig. 10(a) and not in Fig. 10(b). This is as expected from the range of influence of $\kappa$ (Fig. 5) and for the particular $E(y)$ profiles. The near-zero $E(y)$ near the front side attenuates $q(t)$ at short times [Eq. (14)].

\section{EXPERIMENTAL SAMPLES}

Commercial Kapton ${ }^{\mathrm{TM}}$ film Type $30 \mathrm{HN}$, manufactured by the Dupont Co., Wilmington, Delaware, ${ }^{58}$ was originally selected for the experiments as a candidate reference material with supposedly "known" electrical and thermal properties. Its high glass transition temperature $\left(>300^{\circ} \mathrm{C}\right)$ seemed favorable for negligible charging at room temperature under moderate bias voltages. Our results disclosed unexpected electrical behavior that severely tested our numerical methods. The results provide information about the behavior of this polyimide.

Film samples were prepared in pairs bonded to individual substrates. This allowed application of only positive or negative voltage to any one sample. TP measurements before applying a voltage to either sample provided "virgin" data as reference. Substrates were $13 \mathrm{~mm}$ in diameter, flat-faced, oxygen-free copper cylinders. A silver-filled epoxy ${ }^{58}$ (Duralco $120^{\mathrm{TM}}$ from Cotronics Corp., Brooklyn, NY) was used as adhesive. The adhesive formed a thermally resistive layer 


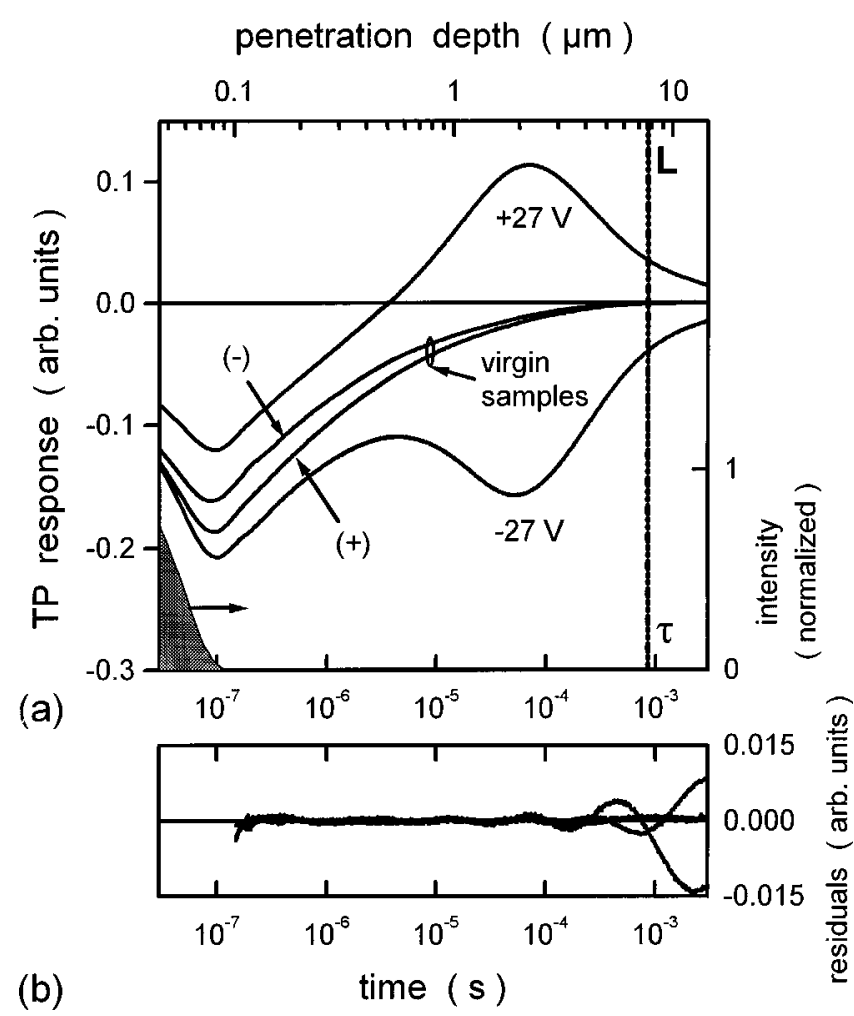

FIG. 11. (a) Measured response $q(t)$ for two PI samples before bias voltage applied (virgin samples) and after $+27 \mathrm{~V}$ was applied to sample that gave $(+)$ virgin curve and $-27 \mathrm{~V}$ was applied to the other that gave the $(-)$ virgin curve for two days at room temperature. Shaded area outlines tail of laser pulse. Upper scale: depth $\delta$ $=(2 D t)^{1 / 2}$, for $D=3.6 \times 10^{-8} \mathrm{~m}^{2} / \mathrm{s}(\delta=L$ at $t=\tau)$. (b) Residuals.

making $T(t, y=L)$ not quite zero as in Fig. 1. The sample thickness was $L=7.5 \pm 0.1 \mu \mathrm{m}$ based on ten measurements at random positions over the electroded region using a precision mechanical gauge. The nominal electrode thickness was $227 \mathrm{~nm}$ as read from a Sloan thickness monitor ${ }^{58}$ at the end of each evaporation. The electrodes, substrate, and laser beam had a common diameter of $13 \mathrm{~mm}$, while the unelectroded part of the samples was around $25 \mathrm{~mm}$. Guided by measurements of the thermal properties of vacuum-deposited $\mathrm{Al},{ }^{62}$ we used $D_{e}=7.1 \times 10^{-5} \mathrm{~m}^{2} / \mathrm{s}$ (a reduction of $8 \%$ from the bulk value) and $\kappa_{e}=100 \mathrm{~W} / \mathrm{mK}$ (a reduction of $40 \%$ from the bulk value).

\section{EXPERIMENTAL RESULTS}

TP responses $q(t)$ were measured in laboratory air $\left(24^{\circ} \mathrm{C}\right.$, $40 \%$ relative humidity). As shown in Fig. 1, $q(t)$ is the charge flowing from the front electrode to the rear electrode in an external short circuit comprising a charge amplifier and a battery used as a bias voltage source. Positive (or negative) bias means that the rear electrode is connected to the positive (or negative) terminal of the battery, the other terminal being connected to system ground.

\section{A. TP responses}

Figure 11(a) shows TP responses $q(t)$ from two samples before and after voltage biasing. The curves labeled "virgin samples" were obtained before the samples had any biasing

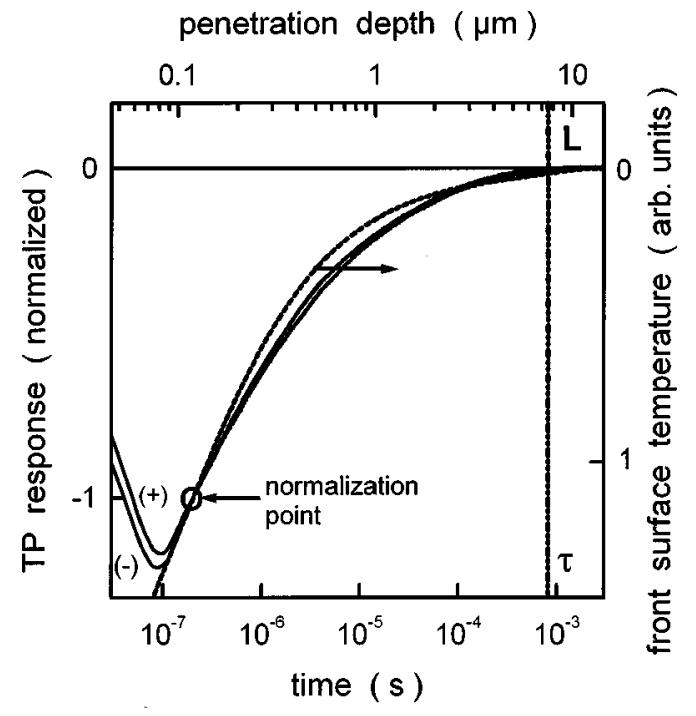

FIG. 12. Comparison of response $q(t)$ for the two virgin samples (solid curves in Fig. 11) and front-surface temperature calculated from Eqs. (4) and (5) (dotted line). All curves normalized to common value at $t=200 \mathrm{~ns}$.

history. The curves " $+27 \mathrm{~V}$ " and " $-27 \mathrm{~V}$," belonging to the samples that gave the virgin curves " + ", and " - ," respectively, were obtained after the samples had been under +27 and $-27 \mathrm{~V}$, respectively, for two days at room temperature. This biasing time was needed for the samples to reach a nominal charging steady state, as judged by the nearcessation of evolution in the response.

The shaded curve at the bottom left of Fig. 11(a) shows the trailing part of a Gaussian fitted to the thermal pulse measured by a photodiode and sampled at $5 \mathrm{~ns}$ intervals. The Gaussian width was $70 \mathrm{~ns}$ with a standard deviation of 0.5 ns. The time $t=0$ was set at the peak of the Gaussian located $30 \mathrm{~ns}$ before the beginning of the time range shown. The steepest part of $q(t)$ inside the thermal pulse should be interpreted only qualitatively because of a $30 \mathrm{~ns}$ preamplifier rise time.

The horizontal axis on the top of Fig. 11(a) shows the thermal penetration depth $\delta$, calculated from the time scale at the bottom using $\delta=(2 D t)^{1 / 2}$ with $D=3.6 \times 10^{-8} \mathrm{~m}^{2} / \mathrm{s}$, a value consistent with results given below (Sec. VB). This yields a thermal transit time of $\tau=0.78 \mathrm{~ms}$. Figure 11(b) shows residuals to be discussed below (they are shown as part of Fig. 11 for convenience).

The observation of a reproducible zero-bias response from each virgin sample unambiguously indicates a stable and unexpected $E(y)$ of internal origin. As previously noted, ${ }^{3}$ when $E(y)$ comes from a charge singularity near the origin represented by a $\delta$ function at $y=0$, the response given by Eq. (14) becomes proportional to the transient temperature of the front surface $T(t, y=0)$. Figure 12 shows the zero-bias virgin $q(t)$ along with the calculated $T(t, y=0)$. All curves are arbitrarily normalized to a common value at $t=200 \mathrm{~ns}$. A strong similarity between the two virgin $q(t)$ and $T(t, y=0)$ is clearly evident, indicating a sharp feature in $E(y)$ independent of any deconvolution procedure.

Figure 13 shows measured responses $q_{0}(t)$ and $q_{1}(t)$ for a ring-mounted film sample (both sides TP accessible), for the TP applied to sides $y=0$ and 1 , respectively. The two 


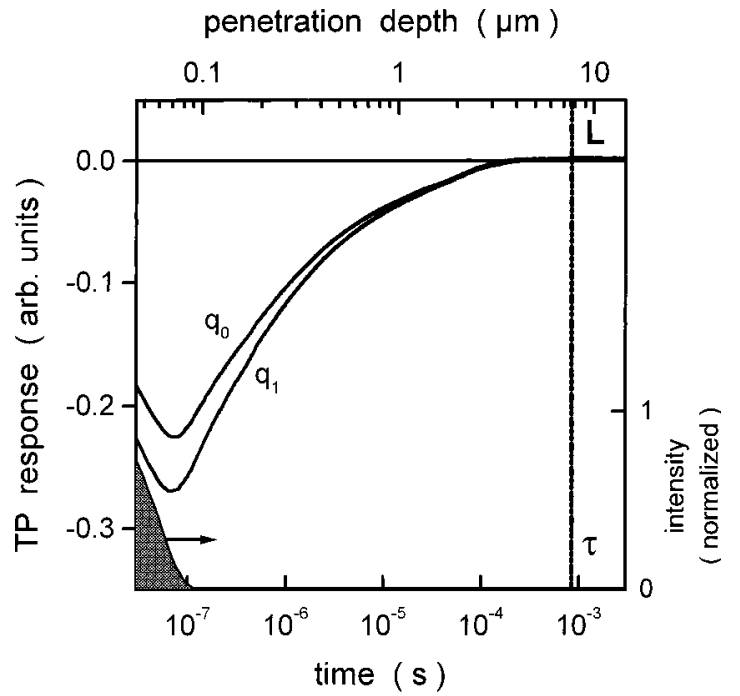

FIG. 13. TP responses $q_{0}(t)$ and $q_{L}(t)$ of ring-mounted virgin sample (free standing, air on both sides) with thermal pulse applied to $z=0(175 \mathrm{~nm} \mathrm{Al})$, upper curve, and $z=L(157 \mathrm{~nm} \mathrm{Al})$, lower curve. Shaded area, tail of thermal pulse.

responses are similar. Small differences are attributed to variations in electrode thickness $(175 \mathrm{~nm}$ at $y=0$ and 157 nm at $y=1)$. Both $q_{0}(t)$ and $q_{1}(t)$ approach zero asymptotically at long times $(t \gg \tau)$ when $T(t, y)$ approaches spatial homogeneity. This zero homogeneous pyroelectric response is a well-known indication ${ }^{4}$ that $q(t)$ comes from charge rather than dipolar polarization (unless the polarization happened to have a zero mean). These measurements suggest that charge layers exist against both surfaces of a foil sample before, and presumably after, it is glued to a substrate. From Eq. (14), when a charge layer is at the sample/substrate interface, it is not expected to be easily observable (for example, in Fig. 12) because (i) the thermally sunk interface makes $T(t, y \approx 1) \approx 0$ at all times, and (ii) the decreasing thermal gradient makes the spatial resolution ${ }^{4,63,64}$ decrease with time and hence depth.

The four response curves in Fig. 11(a) have similar shapes at short times $(t<3 \mu \mathrm{s})$ where the lack of sign change for $\pm 27 \mathrm{~V}$ bias indicates strong binding of the charge layer. Thermal stability tests also showed strong binding of the charge layer (annealing a virgin sample for two hours at $200{ }^{\circ} \mathrm{C}$ reduced the TP response by a factor of 3 but did not eliminate it).

Figure 14 shows difference curves, $\Delta(+)=q(+27 \mathrm{~V})$ $-q(+)$ and $\Delta(-)=q(-27 \mathrm{~V})-q(-)$, obtained from Fig. 11(a). The sign of these two curves clearly depends on the sign of the bias. The horizontal parts at early times come from the electrode-charge term proportional to $\pm C V$. This term is expected to decay when $t \geqslant \tau$ as heat goes into the substrate, but this decay is masked by the broadly peaked parts of $\Delta(+)$ and $\Delta(-)$ with peaks around $t \approx 10^{-4} \mathrm{~s}$. These parts are attributed to charge injection from the substrate (positive charges for positive bias, negative charges for negative bias). The above observations indicate that the TP response has the expected bias-voltage-dependent contributions, in addition to the bias-insensitive near-surface-charge contribution.

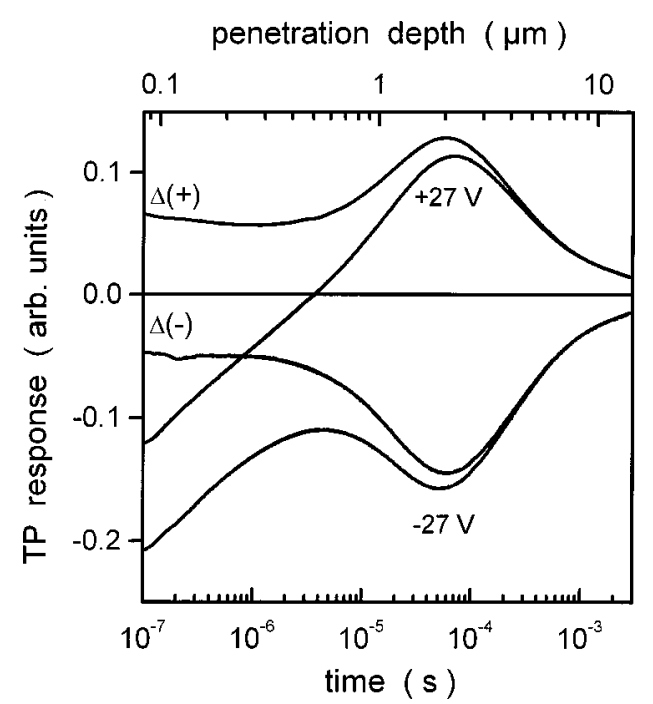

FIG. 14. Difference responses (virgin response removed) $\Delta(+)=q(+27 V)-q(+)$ and $\Delta(-)=q(-27 V)-q(-)$, from Fig. 11. Curves $q(+27 V)$ and $q(-27 V)$ shown for reference. Note separability into capacitive and injected parts.

\section{B. Spatial distributions}

The time range chosen for deconvolution was from $t_{\min }$ $=150 \mathrm{~ns}$ to $t_{\max }=2 \mathrm{~ms}$, as shown graphically by the range of the residuals in Fig. 11(b). This range was sampled by 1700 points (the points up to $4 \mu$ s were $5 \mathrm{~ns}$ apart while the rest were acquired at logarithmically equally spaced intervals). The first usable grid point inside the sample was $y_{2} L$ $\approx 30 \mathrm{~nm}$ (see Sec. IIC 3). A noise amplitude of $\sigma \approx 0.3 \%$ was found by the deconvolution program [Eq. (20)], justifying the value used in the simulations. The negative sign of the determined charge layer is consistent with the negative sign for $\alpha_{c} C$ in Eq. (14), as expected physically and from manufacturer's data. 59

Figure 15 shows deconvolution results obtained from the TP responses in Fig. 11(a). The smallness of the residuals shown in Fig. 11(b) indicates good fits over nearly four orders of magnitude in time. The increasing residuals for $t$

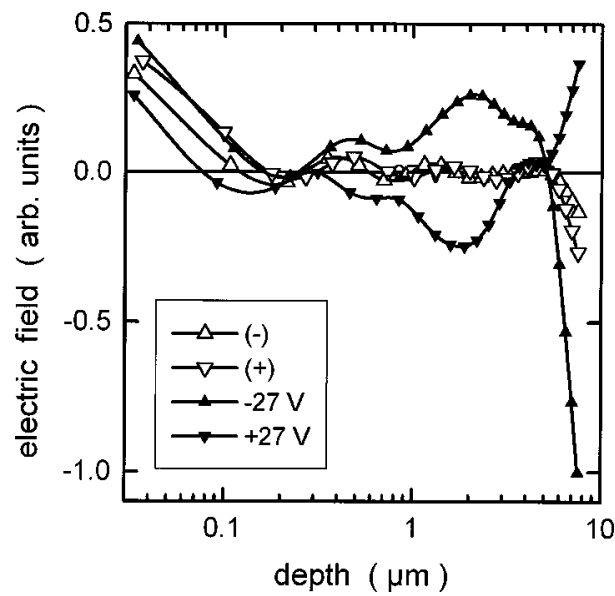

FIG. 15. Field profiles determined from Fig. 12(a) by deconvolution: $(\nabla, \boldsymbol{\nabla})$ from $(+)$ virgin curve and $(+27 \mathrm{~V})$ curve, respectively; $(\triangle, \mathbf{\Lambda})$ from $(-)$ virgin curve and $(-27 \mathrm{~V})$ curve, respectively. Statistical error bars, same size as symbols near front surface, three times larger near rear surface (omitted for clarity). 
$>0.3 \mathrm{~ms}$ are attributed to subtle effects like those suggested in Sec. III E 2 (last paragraph) or from inadequacies in our model. The logarithmic depth scale was chosen in Fig. 15 to spread out the near-surface zone that contains the sharp charge layer. Note that the slope $d E / d(\ln z)$ is proportional to $z$ times the volume density of space charge $\rho(z)$.

\section{Virgin samples (no bias voltage)}

The virgin samples (open triangles in Fig. 15) show a downward-sloping $E(\ln z)$ for $z<200 \mathrm{~nm}$ corresponding to a negative $\rho(z)$ layer of uncertain origin near the front surface. For $200 \mathrm{~nm}<z<5 \mu \mathrm{m}, E(z)$ is nominally zero. The negative dip in $E(\ln z)$ for $z>5 \mu \mathrm{m}$ with a slope indicating negative $\rho(z)$ is believed to be meaningful based on simulations using trapezoidal field profiles ${ }^{64}$ (not shown), which gave the correct sign for the slope of $E(z)$ near the rear side when $E(z)$ went to zero over a distance $<5 \% L$. This suggests that at least the sign of the space charge can be determined in the rear third, in spite of the reduced resolution. ${ }^{64}$ The statistical error bars are approximately the same size as the symbols marking the data points near the front surface and three times larger near the rear side.

To check that a charge layer could not have been a manufacturing artifact, we also performed preliminary TP measurements on $1.7-\mu \mathrm{m}$-thick PL films prepared by spincoating a precursor solution ${ }^{58}$ (Pyralin $^{\mathrm{TM}}$ PI2555) on polished silicon wafers and oven-curing them. Similar frontsurface charge layers were observed.

\section{Samples under bias voltage}

From Fig. 15, it is seen that a bias voltage shifts the field distribution at shallow depth $(200 \mathrm{~nm})$ without noticeably changing the slope. This is consistent with the earlier-noted insensitivity of the bound charge density to an applied voltage. The deeper-lying structure in $E(\ln z)$ that inverts when $U$ is reversed is consistent with charge injection across the interfacial plane. The zero crossings in Fig. 15 indicate penetration of the injected charge to a depth of approximately $2.5 \mu \mathrm{m}$ from the interfacial plane. Hints that positive charge may penetrate deeper into the sample than negative charge could be attributed to the injection being assisted by the field of the front-surface charge.

\section{Thermal parameters}

In the deconvolution, $H_{0}$ was assumed to be $15 \mathrm{~W} / \mathrm{m}^{2} \mathrm{~K}$, typical of ambient air (see Sec. III). By using the iterations described in Sec. III E, an unambiguous minimum was found in the MSE, in spite of the strongly inhomogeneous $E(y)$. The optimal values of the varied parameters corresponding to this minimum are $D_{\text {opt }}=3.6 \times 10^{-8} \mathrm{~m}^{2} / \mathrm{s}, \quad \kappa_{\text {opt }}$ $=0.1 \mathrm{~W} / \mathrm{m} \mathrm{K}$, and $H_{L, \mathrm{opt}}=6 \times 10^{4} \mathrm{~W} / \mathrm{m}^{2} \mathrm{~K}$. The uncertainties based on one standard deviation are $\pm 2 \%$, $\pm 10 \%$, and $\pm 30 \%$, respectively.

The thermal diffusivity $D_{\text {opt }}=3.6 \times 10^{-8} \mathrm{~m}^{2} / \mathrm{s}$ is about half the value $D=7.8 \times 10^{-8} \mathrm{~m}^{2} / \mathrm{s}$ listed in the product data sheet for $25-\mu \mathrm{m}$-thick Kapton ${ }^{\mathrm{TM}}$ films. ${ }^{59}$ The thermal conductivity $\kappa_{\mathrm{opt}}=0.1 \mathrm{~W} / \mathrm{m} \mathrm{K}$ is about the same as the listed value. This makes the ratio $\kappa_{\text {opt }} / D_{\text {opt }}=2.8 \times 10^{6} \mathrm{~J} / \mathrm{m}^{3} \mathrm{~K}$, which is about 1.8 times the heat capacity $\rho c=1.5$ $\times 10^{6} \mathrm{~J} / \mathrm{m}^{3} \mathrm{~K}$ estimated from the product data sheet. ${ }^{59}$ Decreased thermal transport parameters for decreased sample thickness have been reported for other materials, for example, diamond films ${ }^{65}$ and amorphous $\mathrm{SiO}_{2}$ and $\mathrm{Si}_{3} \mathrm{~N}_{4}$ films. ${ }^{66,67}$ Such reductions are generally attributed to an increased influence of inhomogeneities.

From the determined $H_{L, \text { opt }}=6 \times 10^{4} \mathrm{~W} / \mathrm{m}^{2} \mathrm{~K}$ and manufacturer's data on the thermal conductivity of the conductive epoxy, ${ }^{68} \kappa_{\text {glue }}=9.3 \mathrm{~W} / \mathrm{m} \mathrm{K}$, the thickness of the epoxy layer may be estimated. We let $H_{L, \text { opt }}=\kappa_{\text {glue }} / L_{\text {glue }}{ }^{15}$ where $L_{\text {glue }}$ is the thickness of the layer. For a constant temperature gradient in the layer, we obtain $L_{\text {glue }} \approx 150 \mu \mathrm{m}$. The mean of ten measurements made with a precision differential gauge gave a thickness of $160 \pm 20 \mu \mathrm{m}$. The above values are also reasonable from the Ag particle size of 20-50 $\mu \mathrm{m}$ specified by the manufacturer. ${ }^{68}$

\section{DISCUSSION}

\section{A. Data analysis methodology}

Our pyroelectric response equations enable the investigation of both the field/charge profile and the thermal properties of an electroded dielectric film-including the thermal resistance that may couple the film to a thermal sink. In addition to extending profiling to thin films, the analysis provides insights applicable to the study of samples of any thickness. The until now common practice of neglecting electrode effects for thick samples is now seen as a probable root cause of limited analytical resolution achieved in the past (inadequate model). The inclusion of electrode effects is seen, in principle, to allow finding $\kappa$ and $D$ independently of each other (and thus $\rho c=\kappa / D$, ) along with $H_{L}$, in addition to $E(y)$.

When applied to synthetic response data generated using two different assumed $E(y)$ and constant thermal parameters, our data analysis methodology involving regularization, deconvolution, and variation of thermal parameters successfully reproduced the "known" $E(y)$ and thermal parameters. The methodology thus passed our simulation tests. The methodology is expected to work equally well when applied to actual data from samples with homogeneous thermal properties. The possibility of inhomogeneous thermal properties in our samples is raised by the lower-thanbulk value found for $D$, which was interpreted as an effective value weighted strongly by the surface region. This interpretation is strengthened by the argument below in the limit of a singular $E(y)$.

Surface sensitivity of $q(t)$. The surface sensitivity imparted by a singularity in $E(y)$ and its consequences in the determination of the thermal properties may be explored in Eq. (14) by rewriting $T(t, y)$ with an expanded argument $T[t, y ; \kappa(y), D(y)$, etc. $]$ that shows $y$ dependence in the thermal properties [the semicolon is used to separate the independent variables $(t, y)$ from the parameters $(\kappa, D$, etc.) upon which $T$ also depends according to Eq. (4)]. When $E(y)$ is represented by a Dirac $\delta$ function at the front surface, Eq. (14) becomes

$$
q(t) \propto T[t, y=0 ; \kappa(y=0), D(y=0), \text { etc. }] .
$$


This trivial though rigorous result shows that only the surface values of $\kappa$ and $D$ appear in $q(t)$.

In our samples, $q(t)$ is thus expected to depend on thermal properties representative of the shallow depth of the high-field region $(\leqslant 200 \mathrm{~nm})$, much smaller than the film thickness of $7.5 \mu \mathrm{m}$.

\section{B. Electrical properties of polyimide films}

Stable near-surface negative charge layers were established in our samples by visual examination of TP data $[q(t) \propto T(t, y=0)]$ as well as by the more elaborate deconvolution procedure. A chemical origin involving water seems plausible. Water is a by-product of the imidization reaction and cured polyimide is known to take up a small percentage of water from a moist environment. The dissociation of nearsurface water molecules by hydrolysis and partial oxidation of the surface regions are suspected mechanisms for the development of a chemically bound negative charge layer.

The effects of bound and injected charges on the electrical properties of polyimide films may be important in hightechnology applications of this material, as, for example, in microelectroncs where space-charge fields (estimated of order $10 \mathrm{~V} / \mu \mathrm{m}$ ) could exist in an environment of field-effect devices. More fundamentally, an expected frequencydependent response of the inhomogeneously distributed charges could lead to a spatially varying complex dielectric permittivity and make the effective permittivity of a film dependent on its thickness.

\section{Thermal properties of polyimide films}

From Eq. (27), it appears that our smaller-than-bulk value for $D$ is an effective value strongly weighted by the surface region. Since our value for $\kappa$ agrees with the bulk value, $\kappa$ is presumed homogeneous and the low value of $D$ is attributed to an enhanced heat capacity $(\kappa / D)$ near the surface. The enhancement could be attributed simply to bound water (the enhanced value lies between the values for bulk polyimide and water) with a contribution related to the chemical modification associated with binding. Finally, a polaron model of charge trapping ${ }^{69}$ suggests that a contribution to the heat capacity could come from the bound charge layer.

\section{ACKNOWLEDGMENTS}

The authors thank NIST colleagues Dr. B. Dickens for the binary transfer program, Dr. G. T. Davis, Dr. Wen-li Wu, Dr. F. I. Mopsik, Dr. Dan Josell, and Dr. Albert Feldman for careful reading of the manuscript and valuable comments, Alan Band for electronic design, and Dr. A. N. Heckert for assistance in using the NIST computing facilities. The financial support of P. B. by the Deutsche Forschungsgemeinschaft (Contract Nos. BL376/2-1,2) and the U.S. NIST is also gratefully acknowledged.
*Present address: Kunststoff-Zentrum in Leipzig gGmbH, ErichZeigner-Allee 44, D-04229 Leipzig, Germany; Email: bloss@ kuzleipzig.de

†Email address: aime.dereggi@nist.gov

${ }^{1}$ G. M. Sessler, Electrets, 2nd enlarged ed. (Springer-Verlag, Berlin, 1987).

${ }^{2}$ R. E. Collins, J. Appl. Phys. 47, 4804 (1976).

${ }^{3}$ A. S. DeReggi, C. M. Guttman, F. I. Mopsik, G. T. Davis, and M. G. Broadhurst, Phys. Rev. Lett. 40, 413 (1978).

${ }^{4}$ F. I. Mopsik and A. S. DeReggi, J. Appl. Phys. 53, 4333 (1982).

${ }^{5}$ Siegfried Bauer, Phys. Rev. B 47, 11049 (1993).

${ }^{6}$ H. Amjadi and G. M. Sessler, IEEE Report No. 95 CH35842, 1995 (unpublished), p. 532.

${ }^{7}$ P. Bloß, A. S. DeReggi, and H. Schäfer, IEEE Report No. 96 CH35985, 1996 (unpublished), p. 721.

${ }^{8}$ P. Bloß, A. S. DeReggi, and H. Schäfer, in Electronic Packaging Materials Science IX, edited by S. K. Groothuis, P. S. Ho, K. Ishida, and T. Wu, Mater. Res. Soc. Symp. Proc. No. 445 (Materials Research Society, Pittsburgh, 1997), p. 179.

${ }^{9}$ W. J. Parker, R. J. Jenkins, C. P. Butler, and G. L. Abbott, J. Appl. Phys. 32, 1679 (1961).

${ }^{10}$ D. Josell, J. Warren, and A. Cezairliyan, J. Appl. Phys. 78, 6867 (1995).

${ }^{11}$ D. Josell, A. Cezairliyan, D. van Heerden, and B. T. Murray, Int. J. Thermophys. 18, 865 (1997).

${ }^{12}$ K. D. Maglic, A. Cezairliyan, and V. E. Pelettsky, Compendium of Thermophysical Property Measurement Methods, Vol. 1: Survey of Measurement Techniques (Plenum, New York, 1984), p. 305.

${ }^{13}$ K. D. Maglic, A. Cezairliyan, and V. E. Pelettsky, Compendium of Thermophysical Property Measurement Methods, Vol. 1: Recommended Measurement Techniques and Practices (Plenum, New York, 1992), p. 281.

${ }^{14}$ S. Bauer and A. S. DeReggi, J. Appl. Phys. 80, 6124 (1996).

${ }^{15}$ H. S. Carslaw and J. C. Jaeger, Conduction of Heat in Solids, 2nd ed. (Clarendon, Oxford, 1995).

${ }^{16}$ A. N. Tikhonov and V. Y. Arsenin, Solution of Ill-Posed Problems (Wiley, New York, 1977).

${ }^{17}$ J. Honerkamp and J. Weese, Continuum Mech. Thermodyn. 2, 17 (1990).

${ }^{18}$ J. Weese, Comput. Phys. Commun. 69, 99 (1992).

${ }^{19}$ H. Schäfer, U. Albrecht, and R. Richert, Chem. Phys. 182, 53 (1994).

${ }^{20}$ U. Albrecht, H. Schäfer, and R. Richert, Chem. Phys. 182, 61 (1994).

${ }^{21}$ H. Schäfer, B. Mädler, and F. Volke, J. Magn. Reson A 116, 145 (1995).

${ }^{22}$ H. Schäfer and R. Stannarius, J. Magn. Res. B 106, 14 (1995).

${ }^{23}$ H. Schäfer, E. Sternin, R. Stannarius, M. Arndt, and F. Kremer, Phys. Rev. Lett. 76, 2177 (1996).

${ }^{24}$ H. Bauch, R. Böttcher, G. Völkel, A. Pöppel, H. Schäfer, J. Banys, and A. Klöppenpieper, Phys. Rev. B 54, 9162 (1996).

${ }^{25}$ H. Schäfer and E. Sternin, Phys. Can. Mar.-Apr. , 77 (1997).

${ }^{26}$ P. Bloß and H. Schäfer, Rev. Sci. Instrum. 65, 1541 (1994).

${ }^{27}$ P. Bloß, M. Steffen, H. Schäfer, G.-M. Yang, and G. M. Sessler, IEEE Trans. Dielectr. Electr. Insul. 3, 182 (1996).

${ }^{28}$ P. Bloß, M. Steffen, H. Schäfer, G. Eberle, and W. Eisenmenger, IEEE Trans. Dielectr. Electr. Insul. 3, 417 (1996).

${ }^{29}$ P. Bloß, M. Steffen, H. Schäfer, G.-M. Yang, and G. M. Sessler, J. Phys. D 30, 1668 (1997). 
${ }^{30}$ S. B. Lang and D. K. Das-Gupta, Ferroelectrics 39, 1249 (1981).

${ }^{31}$ S. B. Lang, Ferroelectrics 118, 343 (1991).

${ }^{32}$ A. S. DeReggi, M. G. Broadhurst, G. T. Davis, and F. I. Mopsik, IEEE Report No. 87 CH2462-0, 1987 (unpublished), pp. 307, 313.

${ }^{33}$ A. S. DeReggi and J. A. Giacometti, IEEE Report No. 94 CH3456-1, 1994 (unpublished), p. 286.

${ }^{34}$ J. A. Giacometti, A. S. DeReggi, G. T. Davis, B. Dickens, and G. F. Leil-Ferreira, J. Appl. Phys. 80, 6407 (1996).

${ }^{35}$ J. A. Giacometti and A. S. DeReggi, J. Appl. Phys. 24, 3357 (1993).

${ }^{36}$ A. S. DeReggi, B. Dickens, T. Ditchi, C. Alquiè, J. Lewiner, and I. K. Lloyd, J. Appl. Phys. 71, 854 (1992).

${ }^{37}$ A. S. DeReggi and M. G. Broadhurst, Ferroelectrics 73, 351 (1987).

${ }^{38}$ E. Balizer, J. Fedderly, D. Haught, B. Dickens, and A. S. DeReggi, J. Polym. Sci., Part B: Polym. Phys. 32, 365 (1994).

${ }^{39}$ B. Ploss and O. Bianzano, IEEE Report No. 94CH3443-9, 1994 (unpublished), p. 206.

${ }^{40}$ A. Mandelis, Photoacoustic and Thermal Wave Phenomena in Semiconductors (North-Holland, New York, 1987).

${ }^{41}$ H. Grober and S. Erk, Fundamentals of Heat Transfer, 3rd ed. (McGraw-Hill, New York, 1961), p. 174, Table 8.1.

${ }^{42}$ G. F. Leal Ferreira and R. Gerhard-Multhaupt, Phys. Rev. B 42, 7317 (1990).

${ }^{43}$ J. S. Hadamard, Lectures on the Chauchy Problem in Linear Partial Differential Equations (Yale University Press, New Haven, CT, 1973).

${ }^{44}$ A. Morozov, Methods for Solving Incorrectly Posed Problems (Springer, New York, 1984).

${ }^{45} \mathrm{C}$. W. Groetsch, The Theory of Tikhonov Regularization for Fredholm Equations of the First Kind (Pitman, London, 1984).

${ }^{46} \mathrm{~W}$. Groetsch, Inverse Problems in Mathematical Sciences (Vieweg, Braunschweig, 1993).

${ }^{47}$ Inverse and Ill-Posed Problems, edited by H. W. Engl and C. W. Groetsch (Academic, New York, 1986).

${ }^{48}$ V. A. Morozov, Regularization Methods for Ill-Posed Problems (CRC, Boca Raton, FL, 1993).

${ }^{49}$ H. W. Engl, M. Hanke, and A. Neubauer, Regularization of Inverse Problems (Kluwer, Dordrecht, 1996).
${ }^{50}$ A. Tarantola, Inverse Problem Theory (Elsevier, Amsterdam, 1987).

${ }^{51}$ K. Chadan and P. C. Sabatier, Inverse Problems in Quantum Scattering Theory (Springer, New York, 1989).

${ }^{52}$ A. K. Louis, Invers und Schlecht Gestellte Probleme (Teubner, Stuttgart, 1989).

${ }^{53}$ E. Hensel, Inverse Theory and Applications for Engineers (Prentice Hall, Englewood Cliffs, NJ, 1991).

${ }^{54}$ Inverse Problems, edited by S. Kubo (Technical Publications, Atlanta, 1993).

${ }^{55}$ Inverse Scattering Problems in Optics, edited by H. P. Baltes (Springer, Berlin, 1980).

${ }^{56}$ S. W. Provencher, Comput. Phys. Commun. 27, 229 (1982).

${ }^{57}$ J. Jagiello, Langmuir 10, 2778 (1994).

${ }^{58}$ The use of commercial names to identify the materials in no way implies endorsement or recommendation by NIST.

${ }^{59}$ Information sheet supplied by DuPont with the Kapton ${ }^{\mathrm{TM}}$ foils.

${ }^{60}$ Handbook of Chemistry and Physics, 75th ed., edited by D. R. Lide (CRC, Boca Raton, FL, 1994).

${ }^{61}$ R. Emmerich, S. Bauer, and B. Ploss, Appl. Phys. A: Solids Surf. 54, 334 (1992).

${ }^{62}$ The thermal properties of the Al electrodes, $D_{e}$ and $\kappa_{e}$, were determined by J. L. Hostetler in the laboratory of Professor P. M. Norris, University of Virginia; Charlottesville, VA, using a femtosecond laser-pulse technique described in J. L. Hostetler, A. N. Smith, and P. M. Norris, Micro Thermophys. Eng. 1, 237 (1997); Int. J. Thermophys. 19, 569 (1998).

${ }^{63}$ M. Steffen, P. Bloß, and Hartmut Schäfer, IEEE Report No. 94 CH3443-9, 1994 (unpublished), p. 200.

${ }^{64} \mathrm{P}$. Bloß (unpublished).

${ }^{65}$ K. E. Goodson, M. I. Flik, L. T. Su, and D. A. Antoniadis, IEEE Electron Device Lett. 14, 490 (1993).

${ }^{66}$ K. E. Goodson, O. W. Käding, M. Rösler, and R. Zachai, J. Appl. Phys. 77, 1385 (1995).

${ }^{67}$ A. J. Griffin, Jr., F. R. Brotzen, and P. J. Loos, High Temp. Mater. Sci. 33, 217 (1995).

${ }^{68}$ Information sheet (Catalog Vol. 96, No. 29) supplied by Cotronics Corporation (Brooklyn, N.Y.) with the Duralco ${ }^{\mathrm{TM}}$ adhesives.

${ }^{69}$ G. Moya and G. Blaise, in Space Charge in Solid Dielectrics, edited by J. C. Fothergill and L. A. Dissado (Dielectrics Society, University of Leicester, Leicester, England, 1997). 